\title{
A VLBA survey of the core shift effect in AGN jets
}

\section{Evidence of dominating synchrotron opacity $\star$}

\author{
K. V. Sokolovsky ${ }^{1,2}$, Y. Y. Kovalev ${ }^{2,1}$, A. B. Pushkarev ${ }^{1,3,4}$, and A. P. Lobanov ${ }^{1}$ \\ ${ }^{1}$ Max-Planck-Institut für Radioastronomie, Auf dem Hügel 69, 53121 Bonn, Germany \\ e-mail: ksokolov@mpifr-bonn.mpg.de \\ 2 Astro Space Center of Lebedev Physical Institute, Profsoyuznaya Str. 84/32, 117997 Moscow, Russia \\ 3 Pulkovo Astronomical Observatory, Pulkovskoe Chaussee 65/1, 196140 St. Petersburg, Russia \\ ${ }^{4}$ Crimean Astrophysical Observatory, 98409 Nauchny, Crimea, Ukraine
}

Received 4 November 2010 / Accepted 29 March 2011

\begin{abstract}
Context. The effect of a frequency dependent shift of the VLBI core position (known as the "core shift") was predicted more than three decades ago and has since been observed in a few sources, but often within a narrow frequency range. This effect has important astrophysical and astrometric applications.

Aims. To achieve a broader understanding of the core shift effect and the physics behind it, we conducted a dedicated survey with NRAO's Very Long Baseline Array (VLBA).

Methods. We used the VLBA to image 20 pre-selected sources simultaneously at nine frequencies in the $1.4-15.4 \mathrm{GHz}$ range. The core position at each frequency was measured by referencing it to a bright, optically thin feature in the jet.

Results. A significant core shift has been successfully measured in each of the twenty sources observed. The median value of the core shift is found to be 1.21 mas if measured between 1.4 and $15.4 \mathrm{GHz}$, and 0.24 mas between 5.0 and $15.4 \mathrm{GHz}$. The core position, $r_{\mathrm{c}}$, as a function of frequency, $v$, is found to be consistent with an $r_{\mathrm{c}} \propto v^{-1}$ law. This behavior is predicted by the Blandford \& Königl model of a purely synchrotron self-absorbed conical jet in equipartition. No systematic deviation from unity of the power law index in the $r_{\mathrm{c}}(v)$ relation has been convincingly detected.

Conclusions. We conclude that neither free-free absorption nor gradients in pressure and/or density in the jet itself and in the ambient medium surrounding the jet play a significant role in the sources observed within the 1.4-15.4 GHz frequency range. These results support the interpretation of the parsec-scale core as a continuous Blandford-Königl type jet with smooth gradients of physical properties along it.
\end{abstract}

Key words. galaxies: active - galaxies: jets - quasars: general - radio continuum: general

\section{Introduction}

In VLBI images of relativistic AGN jets, the "core" is the most compact feature near the apparent base of the jet. It is identified with the surface at which the optical depth $\tau_{v} \approx 1$ (photosphere) in a continuous jet flow with gradually changing physical properties (Blandford \& Königl 1979). The position of this surface is a function of the observation frequency, $v$, and the exact form of this function depends on the absorption mechanism. The above interpretation of the core is supported by observations of the "core shift" effect serendipitously discovered by Marcaide \& Shapiro (1984) and later investigated by, among others, Zensus et al. (1995), Lobanov (1998), Kovalev et al. (2008a), and O'Sullivan \& Gabuzda (2009). Additional support comes from observations of the related increase in the apparent core size toward lower frequencies (Unwin et al. 1994; Yang et al. 2008).

An alternative interpretation of the VLBI core as a standing shock in the jet is discussed by Marscher (2006, 2008). This interpretation may be relevant for high-frequency observations of some sources. The standing shock model does not predict a

^ Full Fig. 1 is available in electronic form at http: //www . aanda.org shift in the apparent core position with frequency unless one resorts to an assumption that there is a series of multiple standing shocks, and different shocks are observed as the core at different frequencies. In some objects the core could be a region where the jet bends such that it is more aligned with the line of sight leading to an increased Doppler beaming factor relative to jet regions farther upstream (Marscher 2006). It has even been discussed sometimes, that the radio core may not be part of the jet at all.

The most likely absorption mechanism acting in the compact jet is synchrotron self-absorption (Königl 1981). If the jet is freely expanding and there is an equipartition between the particle and magnetic field energy densities, the position $r_{\mathrm{c}}(v)$ of the $\tau_{v}=1$ surface will be $r_{\mathrm{c}}(v) \propto v^{-1}$. If the above assumptions do not hold (e.g., synchrotron self-absorption is not the dominating absorption mechanism), we can expect a different dependency of $r_{\mathrm{c}}(v)$.

Investigation of the core shift effect is important for gaining deeper understanding of the structure and physical conditions in ultracompact AGN jets. It may also provide information about the pressure and density gradients in ambient medium surrounding VLBI-scale jets - the broad-line region (BLR) and the inner part of the narrow-line region (NLR) (e.g., Lobanov 2007). The 
Table 1. The large core shift source sample observed with the VLBA.

\begin{tabular}{cccclcc}
\hline \hline IAU name & Alias & RA (J2000) & Dec $(\mathrm{J} 2000)$ & $z$ & Optical class & VLBA epoch \\
\hline $0148+274$ & & $01: 51: 27.146174$ & $+27: 44: 41.79363$ & 1.26 & QSO & $2007-03-01$ \\
$0342+147$ & & $03: 45: 06.416545$ & $+14: 53: 49.55818$ & 1.556 & QSO & $2007-06-01$ \\
$0425+048$ & OF 42 & $04: 27: 47.570531$ & $+04: 57: 08.32555$ & $0.517^{1}$ & AGN & $2007-04-30$ \\
$0507+179$ & & $05: 10: 02.369133$ & $+18: 00: 41.58160$ & 0.416 & AGN & $2007-05-03$ \\
$0610+260$ & \multirow{2}{*}{ C 154} & $06: 13: 50.139161$ & $+26: 04: 36.71971$ & 0.580 & QSO & $2007-03-01$ \\
$0839+187$ & & $08: 42: 05.094175$ & $+18: 35: 40.99050$ & 1.272 & QSO & $2007-06-01$ \\
$0952+179$ & & $09: 54: 56.823616$ & $+17: 43: 31.22204$ & 1.478 & QSO & $2007-04-30$ \\
$1004+141$ & & $10: 07: 41.498089$ & $+13: 56: 29.60070$ & 2.707 & QSO & $2007-05-03$ \\
$1011+250$ & & $10: 13: 53.428771$ & $+24: 49: 16.44062$ & 1.636 & QSO & $2007-03-01$ \\
$1049+215$ & & $10: 51: 48.789077$ & $+21: 19: 52.31374$ & 1.300 & QSO & $2007-06-01$ \\
$1219+285$ & W Comae & $12: 21: 31.690524$ & $+28: 13: 58.50011$ & 0.1612 & BL Lac & $2007-04-30$ \\
$1406-076$ & & $14: 08: 56.481198$ & $-07: 52: 26.66661$ & 1.493 & QSO & $2007-05-03$ \\
$1458+718$ & \multirow{3}{*}{ C 309.1 } & $14: 59: 07.583927$ & $+71: 40: 19.86646$ & 0.904 & QSO & $2007-03-01$ \\
$1642+690$ & & $16: 42: 07.848505$ & $+68: 56: 39.75639$ & 0.751 & QSO & $2007-04-30$ \\
$1655+077$ & & $16: 58: 09.011464$ & $+07: 41: 27.54034$ & 0.621 & QSO & $2007-06-01$ \\
$1803+784$ & & $18: 00: 45.683905$ & $+78: 28: 04.01839$ & 0.680 & QSO & $2007-05-03$ \\
$1830+285$ & & $18: 32: 50.185622$ & $+28: 33: 35.95514$ & 0.594 & QSO & $2007-03-01$ \\
$1845+797$ & \multirow{2}{*}{ C 390.3 } & $18: 42: 08.989895$ & $+79: 46: 17.12825$ & 0.056 & AGN & $2007-06-01$ \\
$2201+315$ & & $22: 03: 14.975788$ & $+31: 45: 38.26990$ & 0.298 & QSO & $2007-04-30$ \\
$2320+506$ & & $23: 22: 25.982173$ & $+50: 57: 51.96364$ & 1.279 & QSO & $2007-05-03$ \\
\hline
\end{tabular}

Notes. Column 1 - IAU source name (B1950); Col. 2 - commonly used source name; Cols. 3 and 4 - VLBI position, for details see http:// astrogeo.org/vlbi/solutions/rfc_2010c/ and Beasley et al. (2002), Fomalont et al. (2003), Petrov et al. (2005, 2006), Kovalev et al. (2007), Petrov et al. (2008, 2009); Cols. 5 and 6 - redshift and optical class from Véron-Cetty \& Véron (2010); Col. 7 - epoch of multifrequency VLBA observations reported in this paper. ${ }^{(1)}$ Spectroscopic redshift obtained by Afanasiev et al. (2003); ${ }^{(2)}$ photometric redshift, see Finke et al. (2008).

impact of the effect on interpretations of current and future radio Very Long Baseline Interferometry (VLBI) astrometric measurements has been discussed by Rioja et al. (2005) and Porcas (2009). The core shift is expected to introduce a systematic offsets of approximately 0.1 mas between the optical (6000 $)$ and radio $(8 \mathrm{GHz})$ positions of reference extragalactic sources (Kovalev et al. 2008a), which needs to be taken into account for accurate radio-optical reference frame alignment in the era of modern space-based astrometric missions (Lindegren 2007) such as GAIA (Perryman et al. 2001) and for accurate spacecraft navigation with VLBI (e.g., Hildebrand et al. 1994; Sekido et al. 2004; Pogrebenko et al. 2009).

In this paper, we present observational results from a dedicated nine-frequency $(1.4-15.4 \mathrm{GHz})$ VLBA survey of the core shift effect in 20 compact extragalactic radio sources. The selection of targets, the VLBA observation setup and our data reduction strategy are described in Sect. 2. Section 3 presents the core shift measurement technique. In Sect. 4, we discuss the results and their implication for the dominating absorption mechanism in the core region. Section 5 presents a brief summary of this work. Throughout this paper, we adopt a $\Lambda$-CDM cosmology, with the following values for the cosmological parameters: $H_{0}=0.71, \Omega_{\mathrm{m}}=0.27$, and $\Omega_{\Lambda}=0.73$ (Komatsu et al. 2009).

\section{VLBA observations}

\subsection{Sample selection}

A blind survey for sources with measurable core shifts would be an extraordinarily time consuming project because it requires performing simultaneous multifrequency, high-resolution, high dynamic range VLBI observations. Therefore, we turned to an existing large-scale geodetic VLBI database to find promising candidates for the survey. Kovalev et al. (2008a) have imaged and analyzed 277 sources from the Research and Development
VLBA program (RDV, see Fey \& Charlot 1997; Petrov et al. 2009) observations made in 2002-2003. RDV experiments involve simultaneous dual frequency $2.3 / 8.6 \mathrm{GHz}$ ( $S / X$ band) observations with the VLBA and up to ten other globally distributed antennas. The data processing technique and imaging results are described by Pushkarev \& Kovalev (in prep.). The core shift was measured by Kovalev et al. (2008a) by referencing the core position to optically thin jet features, the positions of which are not expected to change with frequency.

During the preparation of the Kovalev et al. (2008a) work, 18 sources with large core shifts between 2.3 and $8.6 \mathrm{GHz}$ were selected for detailed multifrequency study with the VLBA, which we report here. These sources were selected in such a way that they not only show large core shifts in this frequency range, but are also particularly suitable for measuring it by referencing the core position to bright jet features - the technique used in this paper. This procedure is needed because the absolute position information is lost during the phase self-calibration process necessary for VLBI imaging; therefore, it is not known a priori how images at different frequencies should be aligned. Two more sources, 2201+315 and 3C $309.1(1458+718)$, were added to the list in order to continue our previous core shift studies (Lobanov 1998; Ros \& Lobanov 2002; Homan \& Kovalev 2003, priv. comm.).

\subsection{VLBA observation setup}

Twenty sources selected as promising candidates for a detailed core shift investigation were observed with the VLBA (Napier 1994) during four $24 \mathrm{~h}$ sessions in March-June 2007. The list of the observed sources is presented in Table 1. Each source was observed simultaneously using $L, S, C, X$, and $K_{\mathrm{u}}$ band receivers (according to the Institute of Electrical and Electronics Engineers or IEEE nomenclature, see Table 2) in the 1.4-15.4 GHz range. In each band four $8 \mathrm{MHz}$-wide frequency 
K. V. Sokolovsky et al.: A VLBA survey of the core shift effect in AGN jets. I.

Table 2. IF central frequencies.

\begin{tabular}{lrr}
\hline \hline IEEE Band & IF & $\begin{array}{r}\text { Frequency } \\
\text { (MHz) }\end{array}$ \\
\hline$L$ & 1 & 1404.49 \\
$L$ & 2 & 1412.49 \\
$L$ & 3 & 1658.49 \\
$L$ & 4 & 1666.49 \\
& & \\
$S$ & 1 & 2275.49 \\
$S$ & 2 & 2283.49 \\
$S$ & 3 & 2383.49 \\
$S$ & 4 & 2391.49 \\
& & \\
$C$ & 1 & 4604.49 \\
$C$ & 2 & 4612.49 \\
$C$ & 3 & 4999.49 \\
$C$ & 4 & 5007.49 \\
& & \\
$X$ & 1 & 8104.49 \\
$X$ & 2 & 8112.49 \\
$X$ & 3 & 8425.49 \\
$X$ & 4 & 8433.49 \\
& & \\
$K_{\mathrm{u}}$ & 1 & 15353.49 \\
$K_{\mathrm{u}}$ & 2 & 15361.49 \\
$K_{\mathrm{u}}$ & 3 & 15369.49 \\
$K_{\mathrm{u}}$ & 4 & 15377.49 \\
\hline & & \\
& &
\end{tabular}

Notes. Column 1 - Radio band name according to the IEEE radar band nomenclature; Col. 2 - number of the frequency channel (IF); Col. 3 - central frequency of the frequency channel (IF).

channels (IFs, Table 2) were recorded in both right and left circular polarizations with 2-bit sampling and a total aggregate bit rate of $256 \mathrm{Mbits} / \mathrm{s}$. The correlation of the data was performed at the VLBA Array Operation Center in Socorro, NM, USA, with an averaging time of $2 \mathrm{~s}$. Data in $L, S, C$, and $X$ bands were divided into two sub-bands (two IFs in each sub-band) centered at $1.4,1.7,2.3,2.4,4.6,5.0,8.1$, and $8.4 \mathrm{GHz}$, respectively. The $K_{\mathrm{u}}$ band was not divided into sub-bands in order to achieve similar image sensitivity to other frequencies in a comparable integration time. All four IFs in the $K_{\mathrm{u}}$ band were stacked together around $15.4 \mathrm{GHz}$. The central frequencies of the sub-bands were chosen in such a way that at least one sub-band in each band is centered at a frequency for which antenna gain curve measurements are available. A special procedure was applied to ensure accurate amplitude calibration of all sub-bands (Sect. 2.3).

\subsection{VLBA data calibration and imaging}

The initial calibration was conducted in AIPS (Greisen 2003) following the standard VLBA calibration procedure involving (i) a priori amplitude calibration with measured antenna gain curves and system temperatures; (ii) phase calibration using the phase-cal signal injected during observations; and (iii) fringe fitting. The fringe fitting was performed by the task FRING. A separate solution for the group delay and phase rate was made for each frequency channel (IF). As the final step in calibration, bandpass corrections were applied utilizing the task BPASS.

The sources were imaged independently in each band, using the CLEAN algorithm (Högbom 1974) implemented in the Difmap software (Shepherd 1997). Global amplitude corrections for left and right circular polarization for each IF at each antenna were determined by comparing the total intensity CLEAN
Table 3. Amplitude corrections for the BK134 VLBA experiment.

\begin{tabular}{|c|c|c|c|c|c|}
\hline Ant. & Band & IF & Epoch & Polarization & Corr. \\
\hline$\overline{\mathrm{BR}}$ & $L$ & 1 & All & LCP & 1.17 \\
\hline BR & $L$ & 2 & All & LCP & 1.13 \\
\hline LA & $L$ & 1 & All & LCP & 0.89 \\
\hline LA & $L$ & 2 & All & LCP & 0.90 \\
\hline $\mathrm{HN}$ & $L$ & $1-4$ & 2007-06-01 & LCP, RCP & 0.75 \\
\hline OV & $L$ & 1 & All & $\mathrm{RCP}$ & 1.17 \\
\hline OV & $L$ & 2 & All & $\mathrm{RCP}$ & 1.15 \\
\hline $\mathrm{BR}$ & $S$ & 3 & All & LCP & 1.13 \\
\hline LA & $S$ & 1 & All & LCP & 1.12 \\
\hline LA & $S$ & 2 & All & LCP & 1.09 \\
\hline LA & $S$ & 3 & All & LCP & 1.19 \\
\hline LA & $S$ & 4 & All & LCP & 1.23 \\
\hline $\mathrm{HN}$ & $S$ & 3 & All & LCP & 0.90 \\
\hline $\mathrm{HN}$ & $S$ & 4 & All & LCP & 0.89 \\
\hline $\mathrm{KP}$ & $S$ & 1 & All & LCP & 1.09 \\
\hline $\mathrm{KP}$ & $S$ & 2 & All & $\mathrm{LCP}$ & 1.13 \\
\hline NL & $S$ & 3 & All & LCP & 1.09 \\
\hline MK & $S$ & 3 & All & LCP & 1.13 \\
\hline MK & $S$ & 4 & All & LCP & 1.14 \\
\hline LA & $S$ & 1 & All & RCP & 0.85 \\
\hline LA & $S$ & 2 & All & RCP & 0.85 \\
\hline LA & $S$ & 3 & All & RCP & 0.76 \\
\hline LA & $S$ & 4 & All & RCP & 0.79 \\
\hline OV & $S$ & 3 & All & RCP & 0.86 \\
\hline OV & $S$ & 4 & All & $\mathrm{RCP}$ & 0.89 \\
\hline KP & $C$ & 1 & All & LCP & 1.10 \\
\hline KP & C & 2 & All & LCP & 1.11 \\
\hline $\mathrm{BR}$ & $C$ & 1 & All & LCP & 0.91 \\
\hline KP & $C$ & 1 & All & RCP & 1.11 \\
\hline KP & $C$ & 2 & All & $\mathrm{RCP}$ & 1.09 \\
\hline MK & $C$ & 1 & All & RCP & 1.24 \\
\hline MK & $C$ & 2 & All & $\mathrm{RCP}$ & 1.17 \\
\hline OV & $X$ & 1 & All & LCP & 1.21 \\
\hline OV & $X$ & 2 & All & LCP & 1.20 \\
\hline KP & $X$ & 1 & All & RCP & 0.91 \\
\hline KP & $X$ & 2 & All & RCP & 0.93 \\
\hline BR & $K_{\mathrm{u}}$ & 1 & All & LCP & 0.94 \\
\hline BR & $K_{\mathrm{u}}$ & 4 & All & LCP & 0.95 \\
\hline SC & $K_{\mathrm{u}}$ & 1 & All & LCP & 1.05 \\
\hline KP & $K_{\mathrm{u}}$ & 2 & All & RCP & 1.42 \\
\hline
\end{tabular}

Notes. Column 1 - Antenna name; Col. 2 - radio band name; Col. 3 - number of the frequency channel (IF); Col. 4 - epoch to which the amplitude correction was applied; Col. 5 - polarization (right or left circular); Col. 6 - amplitude correction coefficient.

model obtained with the initially calibrated data (again using Difmap). The amplitude corrections obtained were averaged over all the sources observed in the experiment with the exception of $0610+260$ and $1830+285$. These two sources have steep radio spectra (Kovalev et al. 1999, 2002) and were not used for the amplitude corrections computation in order to avoid introducing systematic errors into the corrections. The significant amplitude correction factors that were found are listed in Table 3. These amplitude corrections were introduced into the dataset using the AIPS task CLCOR. After that $L, S, C$, and $X$ band data were split into two sub-bands as described above, and final total intensity imaging for each sub-band was conducted independently using Difmap. Naturally weighted CLEAN images of the 

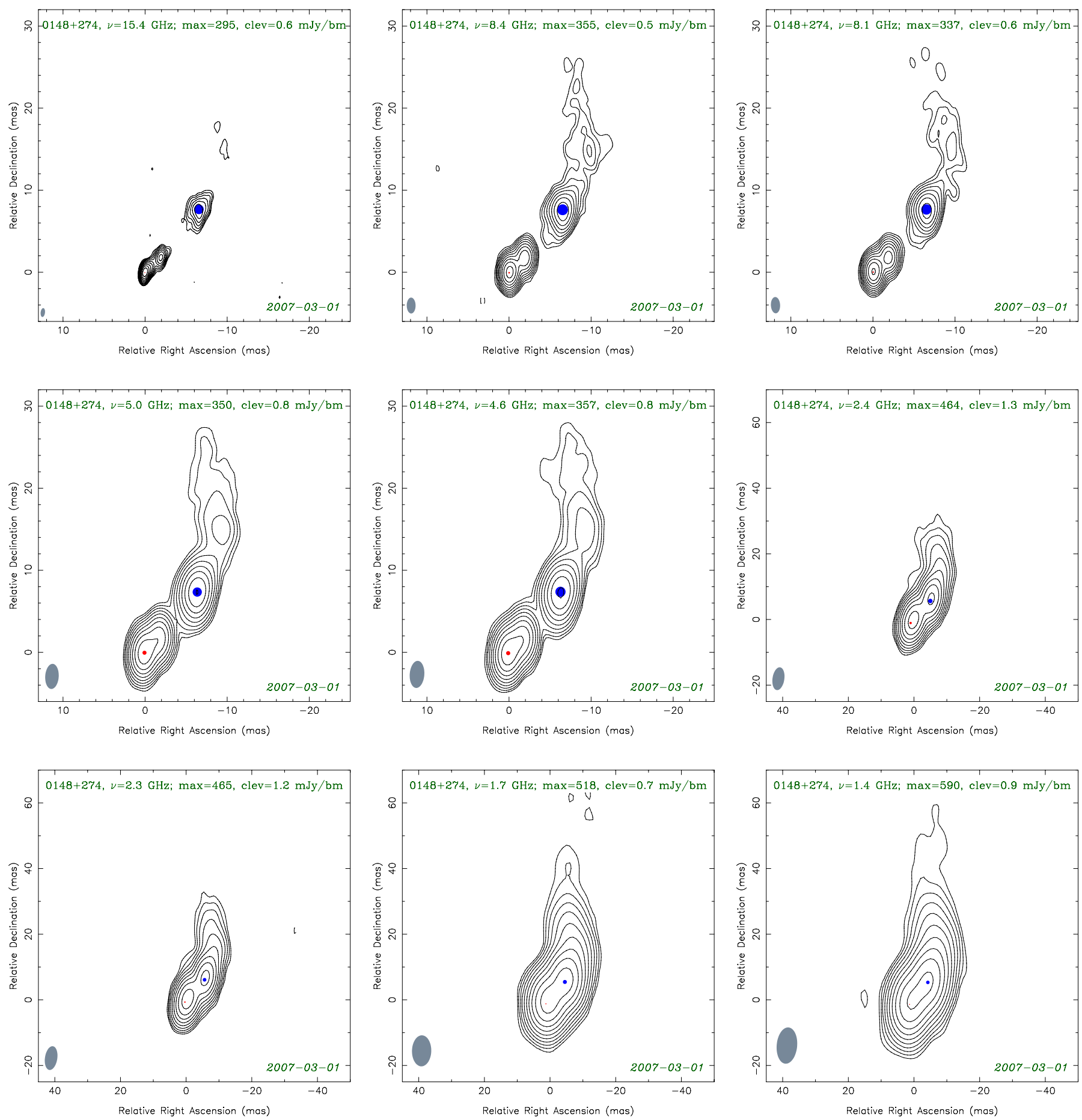

Fig. 1. Naturally weighted CLEAN images of the observed sources between 1.4 and $15 \mathrm{GHz}$. The lowest contour value "clev" is chosen at four times the rms noise, the peak brightness is given by "max". The contour levels increase by factors of two. The dashed contours indicate negative brightness. The beam's full width at half maximum (FWHM) is shown in the bottom left corner of the images in gray. An epoch of observation is shown in the bottom right corner. Red and blue spots indicate the positions and sizes (FWHM) of Gaussian model components for the core and the jet features, respectively.

observed sources between 1.4 and $15.4 \mathrm{GHz}$ are presented in Fig. 1 available in the electronic version of the paper.

The typical VLBA amplitude calibration accuracy in the $1-10 \mathrm{GHz}$ frequency range is estimated to be $\sim 5 \%$ according to the VLBA Observational Status Summary ${ }^{1}$. Kovalev et al. (2005) and Petrov et al. (2008) have confirmed this estimate

${ }^{1}$ http://www.vlba.nrao.edu/astro/obstatus/current/ to be correct for $S, X$, and $K_{\mathrm{u}}$ bands by comparing VLBA integrated flux density with a single-dish flux density measured from 1 to $22 \mathrm{GHz}$ quasi-simultaneously at RATAN-600 (see description of the program in Kovalev et al. 1999, 2002) and the data from the University of Michigan Radio Astronomy Observatory (UMRAO) monitoring program (Aller et al. 1985, $1992,2003)$. This agrees with the VLBA $K_{\mathrm{u}}$ band amplitude calibration accuracy reported by Homan et al. (2002). We checked 
and confirmed the above estimates for this particular dataset by using our RATAN-600 data at $1-22 \mathrm{GHz}$ and VLA measurements at 5 and $8 \mathrm{GHz}$ from the VLA/VLBA Polarization Calibration Program ${ }^{2}$. This check was particularly efficient with our slowly varying calibrator $0923+392$ observed in each of the four VLBA sessions. It has negligible kiloparsec-scale emission, which makes this comparison possible.

\section{Model fitting and core shift measurements}

The structure of each source at each frequency was modeled in the visibility $(u v)$ plane with a number of circular Gaussian components using the Difmap software (Shepherd 1997). A suitable number of components (typically $\sim 6$ ) was used to account for all significantly detected features of the source structure at a given frequency. The components were visually cross-identified between frequencies on the basis of their position with respect to the core. The VLBI core is identified at each frequency as the bright component at the apparent jet base. As pointed out in Sect. 2.1, the target sources were preselected in a way that such an identification would be possible in a wide frequency range for the brightest jet components.

One bright, optically-thin jet component, which could be identified across all (or most of) the observing frequency range, was chosen, and its distance from the core was measured at each frequency. Model component positions and sizes for the core, as well as the jet feature used for this analysis, are indicated in Fig. 1. In some rare cases we were unable to construct a consistent model to allow for accurate and robust component crossidentification with other frequencies. In these cases no model components are shown in Fig. 1, and no data are used in the analysis. Figure 2 shows the distribution of spectral indexes of the reference components, indicating that they are optically thin in the considered frequency range.

The technique of using an optically thin jet component as a reference point for the core position measurement has previously been successfully tested on $S$ / $X$ band RDV data by Kovalev et al. (2008a) in several ways: (i) similar core shift values were obtained referencing to different jet features of the same source; (ii) the core shift for the quasar $1655+077$ agreed within the errors with previous phase-referenced measurements (Homan \& Kovalev 2003, priv. comm.); (iii) two RDV epochs of the quasar $1642+690$ resulted in values being in good agreement. Kovalev et al. (2008a) have also investigated the effect of the different $u v$-coverage resulting in a different resolution (and amount of blending between the core and nearby jet regions) at $S$ and $X$ bands on core shift measurements. The effect was found to be small compared to the core position shift between these bands (see Fig. 5 in Kovalev et al. 2008a).

In addition to that, we performed the following test to look for indications of blending in our $1.4-15.4 \mathrm{GHz}$ results. If significant blending is present, we should measure systematically larger core shifts for sources with jet directions aligned with the position angle (PA) of the elongated VLBA beam. However, no such dependence is found in the data.

To assess the quality of image alignment using a reference jet component, we inspected spectral index maps of the sources. The spectral index maps were computed by constructing a data cube from the images at different frequencies convolved with the same beam (naturally weighted beam corresponding to the lowest frequency) and fitting a power law to the spectrum in each

\footnotetext{
${ }^{2}$ http://www.aoc.nrao.edu/ smyers/calibration/
}

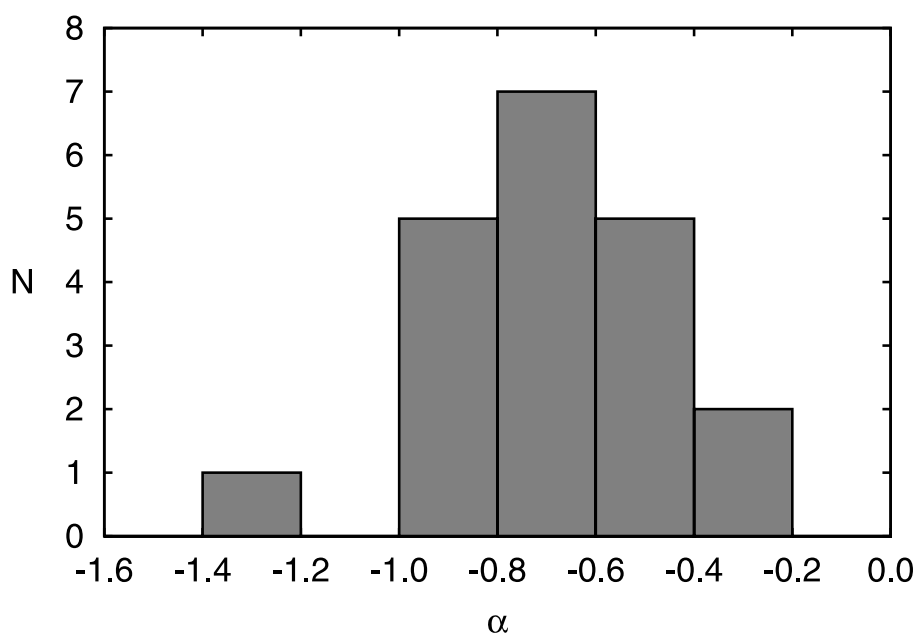

Fig. 2. Distribution of the spectral index ( $\alpha$, flux density $S_{v} \propto \gamma^{\alpha}$ ) of reference jet components. The indexes were computed by fitting a power law to the 1.4-15.4 GHz spectrum. The component flux at each frequency was obtained from the $u v$-model. The source with the steepest reference component spectrum $(\alpha=-1.29)$ is $1011+250$.

individual pixel of the data cube. The power law index (corresponding to the slope of a straight line if plotted in logarithmic scale) is the spectral index. A map with a smooth spectral index gradient along the jet and an absent/weak gradient across the jet would indicate, in general, the correct alignment of images obtained at different frequencies (see, e.g., Marcaide \& Shapiro 1984; Kovalev et al. 2008a). In all cases the spectral index maps revealed no major problems with image alignment, and the core was found to be the only optically thick feature in the jet. The detailed discussion of the spectral imaging results will be presented elsewhere.

An alternative approach to multifrequency VLBI image alignment based on two-dimensional cross-correlation has been proposed by Walker et al. (2000). It has been successfully applied for core shift measurements by O'Sullivan \& Gabuzda (2009) in sources with smooth jets where referencing to a distinct bright jet component was not possible. A potential disadvantage of this method is that, in principle, it may introduce artificial offsets between frequencies in the presence of spectral gradients along the jet. A similar problem may affect the model component-based method used here, if there is a large spectral gradient across a resolved reference component. However, the spectral index gradient is smaller for an individual component than for a large section of parsec-scale jet used in crosscorrelation analysis. Early observations of the frequency dependent difference in distance between the core and jet components were actually interpreted by Biretta et al. (1986) as a sign of spectral gradients across the components. This interpretation is disfavored by the detection of core position shift with frequency in phase-referenced experiments (Marcaide et al. 1985; Rioja et al. 1997; Homan \& Kovalev 2003, priv. comm.) and the fact that strong spectral gradients across individual jet components are not routinely observed if images are aligned correctly.

A significant core shift has been detected in each of the twenty sources observed within our VLBA program (Table 4). The effect is especially pronounced at frequencies below $5 \mathrm{GHz}$. The results are discussed in detail in Sect. 4 and presented visually in Fig. 3.

To statistically estimate typical uncertainty $\sigma_{r}$ of the measured distance $r$ between the core and reference jet component, we used pairs of sub-bands of $X, C, S$, and $L$ bands, assuming 
Table 4. Core shift in milliarcseconds with respect to its position measured at $15.37 \mathrm{GHz}$.

\begin{tabular}{lcccccccr}
\hline \hline & & \multicolumn{7}{c}{ Frequency (GHz) } \\
& 1.41 & 1.66 & 2.28 & 2.39 & 4.61 & 5.00 & 8.11 & 8.43 \\
\hline Name & & & & & & & & \\
$0148+274$ & 1.41 & 1.30 & 1.10 & 1.16 & 0.34 & 0.37 & 0.14 & 0.10 \\
$0342+147$ & 0.71 & 0.64 & 0.30 & 0.28 & 0.07 & 0.07 & 0.03 & 0.06 \\
$0425+048$ & 1.36 & 1.11 & 0.98 & 0.82 & 0.20 & 0.12 & 0.17 & 0.10 \\
$0507+179$ & 0.90 & 0.98 & 0.60 & 0.65 & 0.03 & 0.08 & 0.00 & 0.00 \\
$0610+260$ & 2.20 & 1.99 & 1.53 & 1.58 & 0.45 & 0.50 & 0.21 & 0.22 \\
$0839+187$ & 2.46 & 2.15 & 1.03 & 0.98 & 0.32 & 0.29 & 0.20 & 0.22 \\
$0952+179$ & 1.07 & 1.00 & 0.59 & 0.60 & 0.47 & 0.44 & 0.16 & 0.17 \\
$1004+141$ & 1.61 & 1.21 & & 1.03 & 0.31 & 0.31 & 0.08 & 0.05 \\
$1011+250$ & 1.27 & 1.22 & & & & 0.20 & 0.12 & \\
$1049+215$ & 1.21 & 0.91 & & 0.70 & 0.35 & 0.28 & 0.08 & 0.14 \\
$1219+285$ & & 1.18 & 0.88 & 0.81 & 0.21 & 0.21 & 0.04 & 0.00 \\
$1406-076$ & 0.78 & 0.70 & 0.48 & 0.49 & 0.23 & 0.22 & 0.07 & 0.09 \\
$1458+718$ & 1.39 & 1.25 & 0.98 & 0.94 & 0.18 & & 0.08 & 0.08 \\
$1642+690$ & 1.01 & 0.95 & 0.37 & 0.32 & 0.06 & 0.04 & -0.06 & -0.06 \\
$1655+077$ & 0.88 & 0.66 & & & 0.14 & 0.12 & -0.01 & 0.02 \\
$1803+784$ & 0.60 & 0.49 & 0.48 & 0.46 & 0.04 & 0.04 & 0.09 & 0.01 \\
$1830+285$ & 2.11 & & 0.84 & 0.93 & 0.35 & 0.37 & 0.01 & 0.04 \\
$1845+797$ & & 1.49 & 0.48 & 0.58 & 0.22 & 0.29 & 0.08 & -0.00 \\
$2201+315$ & & & 1.17 & 1.16 & 0.39 & 0.42 & 0.14 & 0.11 \\
$2320+506$ & 0.62 & 0.60 & 0.40 & 0.45 & 0.09 & 0.08 & -0.02 & -0.03 \\
\hline Adopted & & & & & & & & \\
uncertainty & 0.07 & 0.07 & 0.07 & 0.07 & 0.04 & 0.04 & 0.04 & 0.04 \\
\hline
\end{tabular}

Notes. Column 1 - IAU source name (B1950); Cols. 2 to 9 - core shift measured at the given frequency (in milliarcseconds). The last row of the table presents the adopted measurement uncertainty at the given frequency (in milliarcseconds). The adopted measurement uncertainty at $15.37 \mathrm{GHz}$ is 0.04 mas.

that the source structure at these closely separated frequencies is essentially the same and the non-zero difference $\delta r$ between sub-bans in the model fit parameters is determined purely by errors (Fig. 4). This assumption turns out to be correct for $X, C$, and $S$ bands, while at $L$ band a significant core shift between the sub-bands becomes evident. We underline that this approach is possible since data processing of these sub-bands was done completely independently. It should be also noted that the coreshift effect is found to be not significant between the two subbands at $S$-band most probably due to the relatively narrow frequency range (Table 2) achievable at this particular VLBA band. We have also estimated individual errors $\sigma_{r}$ for every source and frequency following two approaches: the one suggested by Fomalont (1999) and its modification by Lee et al. (2008). Then we compared the measured values of $\delta r$ with estimations of its error $\sigma_{\delta r}$ for every sub-band pair. If the $\sigma_{\delta r}$ are estimated correctly following Fomalont (1999) or Lee et al. (2008), we expect that for about $67 \%$ of the sources $\sigma_{\delta r}>\delta r$. We have found out that the Fomalont (1999) method has provided meaningful error estimates for $S, C$, and $X$, while the $L$-bands errors were significantly underestimated due to a very high signal-to-noise ratio of jet components in this band. At the same time, the Lee et al. (2008) method has significantly overestimated the errors in all the bands by $3-40$ times. On the basis of these results, we made a decision to use a statistical method and estimate only a typical $\sigma_{r}$ error for every band. An approach to adopting the same error for each target at a given frequency has an obvious weakness. However, we believe that this turns out to be the most reliable estimate of $\sigma_{r}$ in view of the general problem of calculating the model-dependent error of a component position in VLBI.

In the following, for $X, C$, and $S$ bands we adopt a single typical uncertainty of the distance between the core and reference component, which is the standard deviation of the distance difference measurements made in the two sub-bands of each band, computed using all the sources in our experiment (Fig. 4). For the $X$ band the adopted distance error is $\sigma_{X}=0.04$ mas, for $C$ band $\sigma_{C}=0.04$ mas, and for $S$ band $\sigma_{S}=0.07$ mas. The $K_{\mathrm{u}}$ band has not been divided into two independent sub-bands and for that band we adopt the same position uncertainty as for $X$ band: $\sigma_{K_{\mathrm{u}}}=\sigma_{X}=0.04$ mas. In the $L$ band the mean difference between the distance measured at the lower sub-band $(1.4 \mathrm{GHz})$ and the higher sub-band $(1.7 \mathrm{GHz})$ is $-0.14 \pm 0.03$ mas, which is significantly different from zero. We interpret this as a sign of the core shift effect, which should be more pronounced at lower frequencies, and use the uncertainty derived from the scatter of $S$ band measurements as an estimate of the $L$ band error instead: $\sigma_{L}=\sigma_{S}=0.07$ mas. We note that $\sigma_{L}$ may be underestimated.

\section{Discussion}

\subsection{Typically measured values of the shift}

In the 20 sources observed in our experiment we found the following median values of the core shift with respect to its position at $15.4 \mathrm{GHz}$ : 0.06 mas for $8.4 \mathrm{GHz}, 0.08$ mas for $8.1 \mathrm{GHz}$, 0.22 mas for $5.0 \mathrm{GHz}, 0.22$ for $4.6 \mathrm{GHz}, 0.76$ mas for $2.4 \mathrm{GHz}$, 0.72 mas for $2.3 \mathrm{GHz}, 1.06$ mas for $1.7 \mathrm{GHz}$, and 1.21 mas for $1.4 \mathrm{GHz}$ measurements. See Table 4 for more details. It is difficult to say how applicable these values are to the population of compact extragalactic radio sources as a whole. It is likely that the above values represent a higher extreme of the possible range of values, since the sources with a high core shift were selected for our observations from the list of Kovalev et al. (2008a). The median $S / X$ band core shift value of 0.69 mas obtained for our sample in this study may be compared to the median value of 
K. V. Sokolovsky et al.: A VLBA survey of the core shift effect in AGN jets. I.
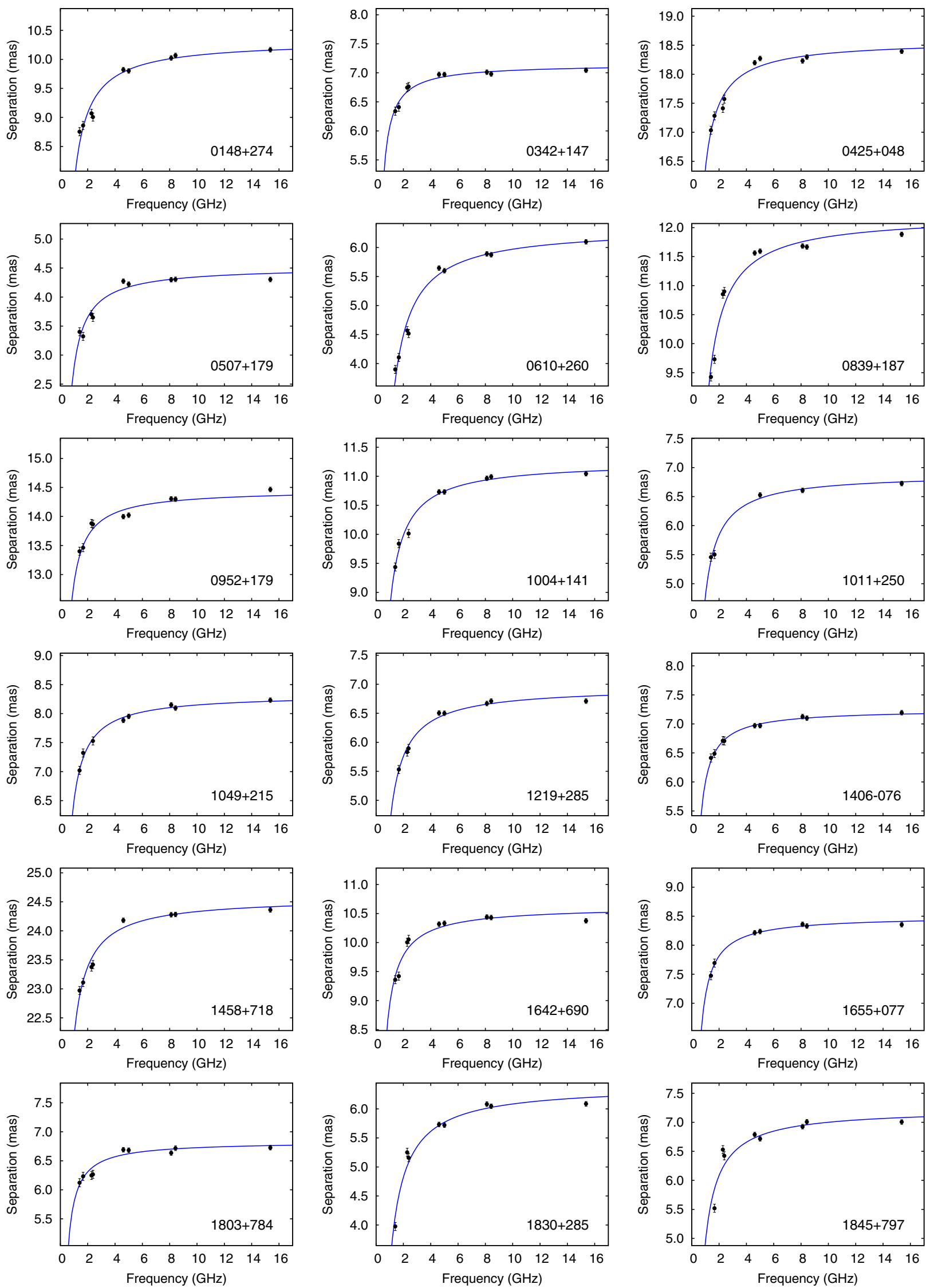

Fig. 3. Separation of the core from a reference optically-thin jet component as a function of frequency. The curve represents the best-fit function $r_{\mathrm{c}}(v)=a+b / v$. The best-fit parameters are presented in Table 5 . 

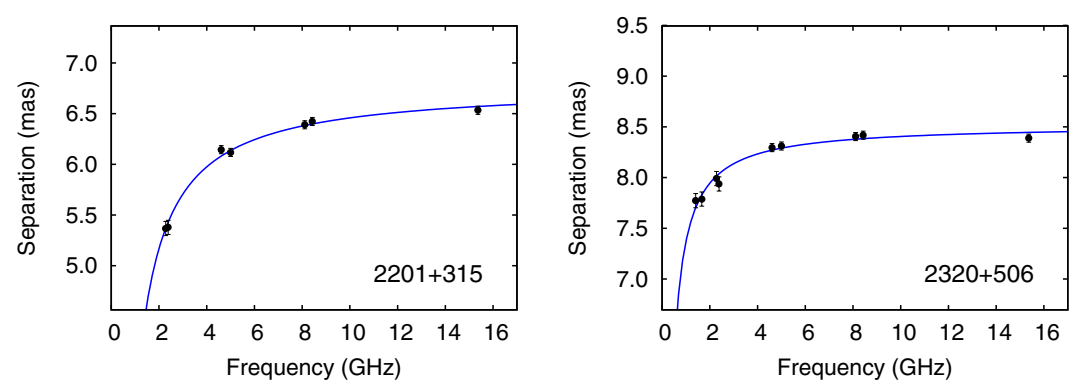

Fig. 3. continued.

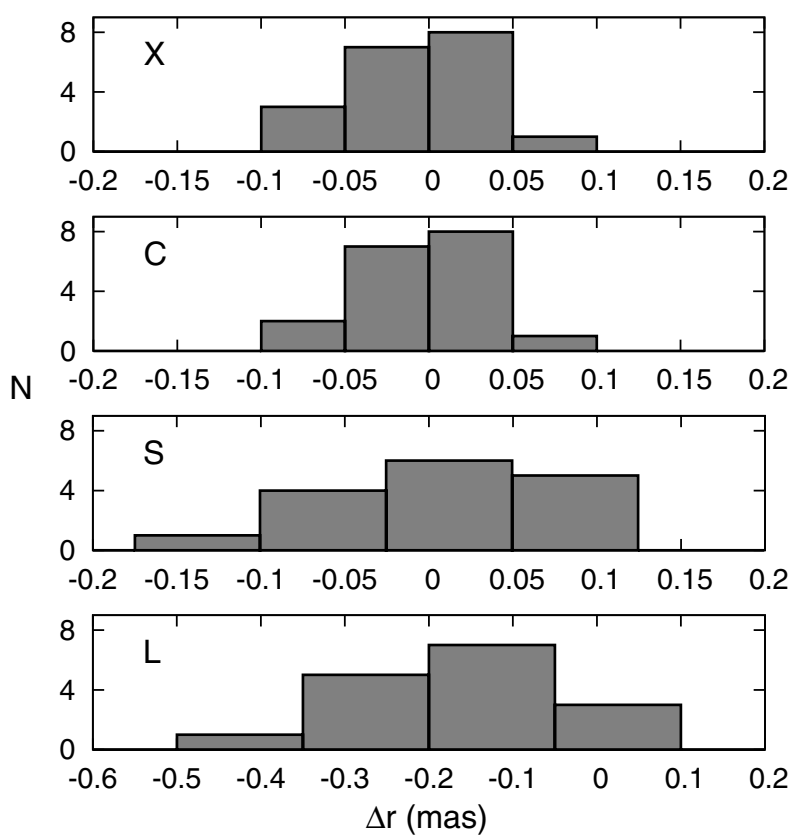

Fig. 4. Distribution of the observed difference in distance between the core and reference component measured at two sub-bands of $X, C, S$, and $L$ bands.

0.44 mas from Kovalev et al. (2008a) for a sample of 29 sources. These samples have 15 sources in common; see more detailed discussion in Sect. 4.5.

O'Sullivan \& Gabuzda (2009) have measured the median core shift of 0.22 mas between 4.6 and $15.4 \mathrm{GHz}$ using a small sample of three objects, none of which is present in our sample. This value is the same as the median $4.6 / 15.4 \mathrm{GHz}$ shift in our sample (0.22 mas). A detailed investigation of the core shift effect in a large complete sample of sources selected not on the basis of previous core shift measurements is needed to derive typical magnitude of the effect in a compact extragalactic radio source.

Finally, we note that misalignment between the parsec-scale jet (resolved in our VLBI observations) and the subparsec scale jet (which we observe as the core) will reduce the frequencydependent distance difference between the core and a reference feature in the parsec-scale jet. Therefore, the sources with the highest measured core shift may be those reflecting the true intrinsic magnitude of the effect.

\subsection{Core position as a function of frequency}

According to Lobanov (1998), the position of the core $r_{\mathrm{c}}(v) \propto$ $v^{-1 / k}$ where the coefficient $k=1$ if (i) the dominating absorption

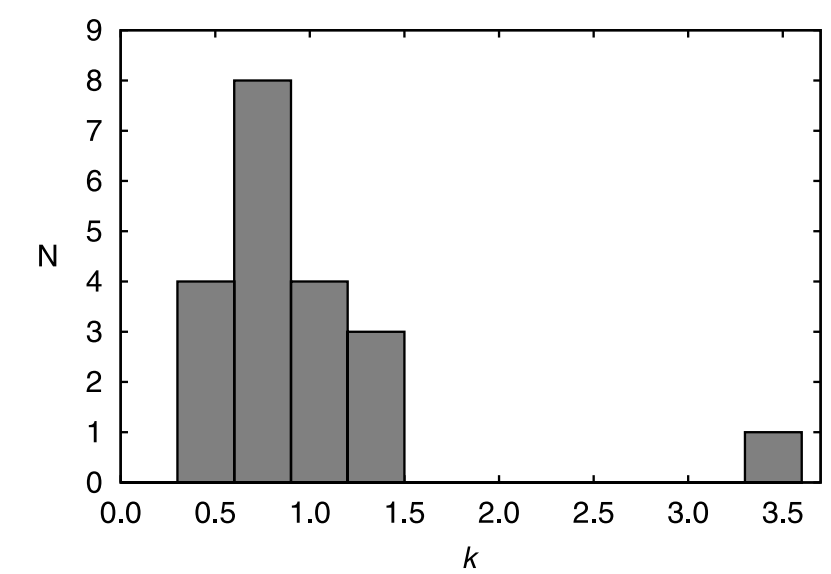

Fig. 5. Distribution of the $k$ parameter in the core position as a function of frequency fit: $r_{\mathrm{c}}(v) \propto v^{-1 / k}$. The mean value is $k=0.99 \pm 0.14$, while the median is 0.82 . One discrepant measurement on the histogram corresponds to the source $0952+179$ where the value of $k$ is not well constrained: $k=3.4 \pm 2.7$.

mechanism is synchrotron self-absorption; (ii) the jet has a conical shape; and (iii) is in equipartition. These assumptions must hold for the ultracompact jet region that we observe as the VLBI core and does not need to be correct all the way along the extended parsec-scale jet.

To test the above assumptions, we fitted the observed distances between the reference jet component and the core in each source with the function $r_{\mathrm{c}}(v)=a+b v^{-1 / k}$ leaving the coefficients $a, b$, and $k$ as free parameters. The Levenberg-Marquardt algorithm (e.g., Press et al. 2002) was used to perform the nonlinear least-squares fit. Figure 5 shows the distribution of the estimated $k$ values. The mean value of the distribution is $k=0.99 \pm 0.14$. This suggests that, for a typical source in the sample in the frequency range of our observations, the above assumptions about the structure of the inner jet (which we observe as the VLBI core) are generally correct. Specifically, we conclude that our observations are consistent with synchrotron self-absorption being the dominating opacity mechanism acting in parsec-scale core regions in the $\mathrm{GHz}$ frequency range.

It should be noted that, since the core shift effect is most pronounced at lower frequencies, the best-fit values of $k$ obtained heavily depend on the $L$-band measurements. At the same time, $L$-band data are also more likely to be affected by blending, an effect that could interfere with the core shift measurements (Kovalev et al. 2008a). To test how sensitive our conclusions are to the $L$-band results, we have repeated the above fitting procedure excluding all $L$-band data. The resulting values of $k$ are on average $30 \%$ lower than to those obtained with $L$-band data included. Naturally, a larger uncertainty is associated with the 
values of $k$ obtained without $L$-band data. The mean values of the two distributions of best-fit $k$ values are consistent at the $2 \sigma$ level.

The values of $k$ presented in Fig. 5 are similar to those obtained for $1038+528$ A by Marcaide \& Shapiro (1984), Marcaide et al. (1985), 3C 345 by Lobanov (1998), 3C 309.1 by Ros \& Lobanov (2002), 0850+581 by Kovalev et al. (2008b), $1418+546,2007+777$, BL Lac by O'Sullivan \& Gabuzda (2009), and Mrk 501 by Croke et al. (2010). These values also agree with the results of Yang et al. (2008), who estimates $k$ from a statistical analysis of parsec-scale core sizes of $\sim 3000$ sources. The core size is expected to be directly related to the observed position of the core along the conical jet (Unwin et al. 1994). The value of $k<1$ was obtained from analysis of the core size in 3C 345 measured between 5 and $22 \mathrm{GHz}$ by Unwin et al. (1994). Values of $k$ slightly more than unity were estimated for BL Lac by analyzing time lags between radio lightcurves obtained at different frequencies (Bach et al. 2006). We also note that Lobanov (1998) has found an indication that $k$ changes with frequency up to values greater than 1 for the quasar 3C 309.1. Kadler et al. (2004) found much higher values of $k$, indicating free-free absorption in the radio galaxy NGC 1052. The key difference between NGC 1052 and the sources observed in our sample is that this radio galaxy shows a two-sided parsec-scale jet. The inner part of the receding jet of NGC 1052 is likely to be obscured by a circumnuclear torus (Kadler et al. 2004).

\subsection{Can the same function describe shifts at all frequencies between 1.4 and $15.4 \mathrm{GHz}$ ?}

To search for a possible change of $k$ with frequency, following Lobanov (1998), we introduce a measure of core position offset

$\Omega_{r v} \propto \frac{\Delta r_{\text {mas }}}{v_{1}^{-1 / k}-v_{2}^{-1 / k}}$

between two frequencies $\left(v_{1}, v_{2}>v_{1}\right)$, where $\Delta r_{\text {mas }}$ is the difference in the apparent core positions at $\nu_{1}$ and $\nu_{2} . \Omega_{r v}$ can be used for assessing the possible variations of $k$. If two values $\Omega_{r v 1,2}$ (measured between frequencies $v_{1}$ and $v_{2}$ ) and $\Omega_{r v 2,3}$ (between $v_{2}$ and $v_{3}$ ) are different, the relation between the corresponding $k_{1,2}$ and $k_{2,3}$ is

$k_{2,3} \simeq k_{1,2} \frac{\log \Omega_{r v 1,2}}{\log \Omega_{r v 1,3}}$

Assuming $k_{1,2}=1$ for the lowest pair of frequencies $\left(v_{1}=\right.$ $1.408 \mathrm{GHz}$ and $v_{2}=1.662 \mathrm{GHz}$ for most cases), we derive the values of $k_{2,3}, k_{2,4}$, and so on. If the assumption about $k=1$ at $L$ band is not correct, that would affect the resulting values of $k$; however, the test will still be sensitive to relative changes in $k$ between frequencies. The obtained values of $k$ were consistent with $k=1$ at a $3 \sigma$ level. Therefore we found no significant changes in $k$ with frequency.

The applicability of the $r_{\mathrm{c}} \propto v^{-1 / k}$ relation to many extragalactic sources has important consequences for the radiooptical reference frame alignment. In the particular case of $k=1$, if the radio source is strongly core-dominated, its position measured using the group delay technique at any frequency (such as those currently used to define the the International Celestial Reference Frame, ICRF, Ma et al. 1998; Fey et al. 2004, and ICRF2, Fey et al. 2010) corresponds to the jet base, not to the radio core position at a given frequency (Porcas 2009). This is due to the additional time delay introduced by the core shift across the observing band (Porcas 2009). The optical emission of an AGN jet is expected to originate much closer to the jet base than the radio core position. Therefore, if the structure of the radio source is dominated by the core, there is no need to introduce core shift corrections to the radio source positions determined from group delays before matching them with optical positions. This is not true, however, for radio source positions determined from phase referencing (Porcas 2009). If a radio source has distinct jet components comparable in brightness to the core (such as many sources considered here; Fig. 1), the group delay would not be a good estimator of the jet base position. The source structure needs to be taken into account prior to comparing the radio and optical source positions in such cases.

\subsection{Fitting results assuming opacity due to synchrotron self-absorption}

As discussed above, our observations are consistent with the assumption of synchrotron self-absorption dominated opacity across the frequency range of this VLBI experiment. We repeated the fit of the function $r_{\mathrm{c}}(v)=a+b v^{-1 / k}$ to the observational data, fixing the value of $k=1$, which is our best estimate. The results are presented in Table 5 and Fig. 3. The values of $a$ and $b$ are given in Table 5. The projected core distance in parsecs is

$r_{\mathrm{c}}(v)[\mathrm{pc}]=\frac{D_{\mathrm{A}}}{N_{\mathrm{rad}}}\left(a[\mathrm{mas}]+b[\mathrm{mas} \mathrm{GHz}] \cdot \frac{1+z}{v_{\mathrm{em}}[\mathrm{GHz}]}\right)$,

where $a$ [mas] and $b$ [mas $\mathrm{GHz}$ ] are the coefficients obtained from observations, $D_{\mathrm{A}}$ is the angular size distance to the source in parsecs, $N_{\text {rad }} \simeq 206264800$ is the number of milliarcseconds in one radian, $z$ the source redshift, and $v_{\mathrm{em}}[\mathrm{GHz}]$ the emission frequency in the source frame.

We have investigated deviations of the measured distances from the best-fit curve presented in Fig. 3 for each frequency. In all cases the mean difference between the measurement and the model curve is consistent with zero, and its standard deviation is consistent within a factor of two with the adopted typical measurement uncertainties.

It can be seen from Fig. 3 that, while the $r_{\mathrm{c}}(v) \propto v^{-1}$ law generally provides a good fit to the data over the whole observed frequency range with no systematic deviations, there are indications of possible local deviations. This may be due to local deviations from equipartition or conical shape of the jet. Another possibility is that the scatter in distance measurements at two sub-bands used for error estimation does not reflect the total measurement uncertainty. This may happen if there is a systematic factor that influences distance measurements at both sub-bands in a similar way. However, after a careful review of our analysis procedures we could not identify any such factor.

\subsection{Search for core shift variability}

The new measurements of core shifts between 2.3 and $8.4 \mathrm{GHz}$ may be compared to the previous measurements at similar frequencies obtained using the RDV data by Kovalev et al. (2008a). As expected for a big enough sample of variable sources, the mean difference between the measurements obtained at two epochs (2002 for RDV global VLBI data and 2007 for this VLBA dataset) is consistent with zero: $0.01 \pm 0.08$ mas. The mean absolute value of this difference is $0.22 \pm 0.05$ mas, which is a measure of a typical long-term core-shift variability in the studied sample within the accuracy of our measurements. 
Table 5. Distance of the reference jet component from the core as a function of frequency: fit results $(r=a+b / v)$.

\begin{tabular}{lrrrrr}
\hline \hline Source & $a(\mathrm{mas})$ & $b(\mathrm{mas} \mathrm{GHz})$ & $a(\mathrm{pc})$ & $b(\mathrm{pc} \mathrm{GHz})$ & $\mathrm{PA}^{\circ}$ \\
\hline $0148+274$ & $10.32 \pm 0.05$ & $-2.48 \pm 0.19$ & $86.75 \pm 0.42$ & $-47.11 \pm 3.61$ & -40.9 \\
$0342+147$ & $7.15 \pm 0.03$ & $-1.08 \pm 0.10$ & $61.14 \pm 0.26$ & $-23.61 \pm 2.19$ & -88.5 \\
$0425+048$ & $18.58 \pm 0.06$ & $-2.20 \pm 0.19$ & $115.05 \pm 0.37$ & $-20.66 \pm 1.78$ & -100.7 \\
$0507+179$ & $4.52 \pm 0.05$ & $-1.73 \pm 0.19$ & $24.74 \pm 0.27$ & $-13.41 \pm 1.47$ & -100.3 \\
$0610+260$ & $6.34 \pm 0.06$ & $-3.69 \pm 0.20$ & $41.62 \pm 0.39$ & $-38.28 \pm 2.07$ & -94.5 \\
$0839+187$ & $12.21 \pm 0.09$ & $-3.67 \pm 0.31$ & $102.76 \pm 0.76$ & $-70.18 \pm 5.93$ & 15.2 \\
$0952+179$ & $14.46 \pm 0.06$ & $-1.60 \pm 0.19$ & $123.39 \pm 0.51$ & $-33.83 \pm 4.02$ & -3.5 \\
$1004+141$ & $11.25 \pm 0.04$ & $-2.53 \pm 0.13$ & $90.53 \pm 0.32$ & $-75.47 \pm 3.88$ & 132.2 \\
$1011+250$ & $6.89 \pm 0.05$ & $-2.10 \pm 0.15$ & $58.96 \pm 0.43$ & $-47.37 \pm 3.38$ & -107.3 \\
$1049+215$ & $8.33 \pm 0.02$ & $-1.82 \pm 0.09$ & $70.30 \pm 0.17$ & $-35.33 \pm 1.75$ & 108.0 \\
$1219+285$ & $6.95 \pm 0.04$ & $-2.38 \pm 0.16$ & $19.06 \pm 0.11$ & $-7.58 \pm 0.51$ & 109.8 \\
$1406-076$ & $7.25 \pm 0.01$ & $-1.25 \pm 0.05$ & $61.90 \pm 0.09$ & $-26.61 \pm 1.06$ & -103.5 \\
$1458+718$ & $24.57 \pm 0.05$ & $-2.38 \pm 0.18$ & $192.21 \pm 0.39$ & $-35.45 \pm 2.68$ & 163.7 \\
$1642+690$ & $10.62 \pm 0.05$ & $-1.68 \pm 0.19$ & $78.01 \pm 0.37$ & $-21.61 \pm 2.44$ & -167.0 \\
$1655+077$ & $8.50 \pm 0.02$ & $-1.37 \pm 0.08$ & $57.64 \pm 0.14$ & $-15.06 \pm 0.88$ & -42.9 \\
$1803+784$ & $6.83 \pm 0.04$ & $-1.04 \pm 0.14$ & $48.21 \pm 0.28$ & $-12.33 \pm 1.66$ & -96.0 \\
$1830+285$ & $6.40 \pm 0.06$ & $-3.15 \pm 0.24$ & $42.50 \pm 0.40$ & $-33.34 \pm 2.54$ & -38.5 \\
$1845+797$ & $7.23 \pm 0.09$ & $-2.29 \pm 0.35$ & $7.76 \pm 0.10$ & $-2.59 \pm 0.40$ & -38.0 \\
$2201+315$ & $6.78 \pm 0.03$ & $-3.22 \pm 0.15$ & $29.83 \pm 0.13$ & $-18.39 \pm 0.86$ & -135.3 \\
$2320+506$ & $8.52 \pm 0.03$ & $-1.15 \pm 0.09$ & $71.76 \pm 0.25$ & $-22.07 \pm 1.73$ & -135.8 \\
\hline
\end{tabular}

Notes. Column 1 - IAU source name (B1950); Cols. 2 and 3 - coefficients of the best-fit curve $r=a+b / v$ with their uncertainties; Cols. 3 and 4 - same coefficients converted to the projected linear scale; Col. 5 - median (across all frequencies) position angle of the reference jet component with respect to the core; it marks the direction along which the core shift was measured.

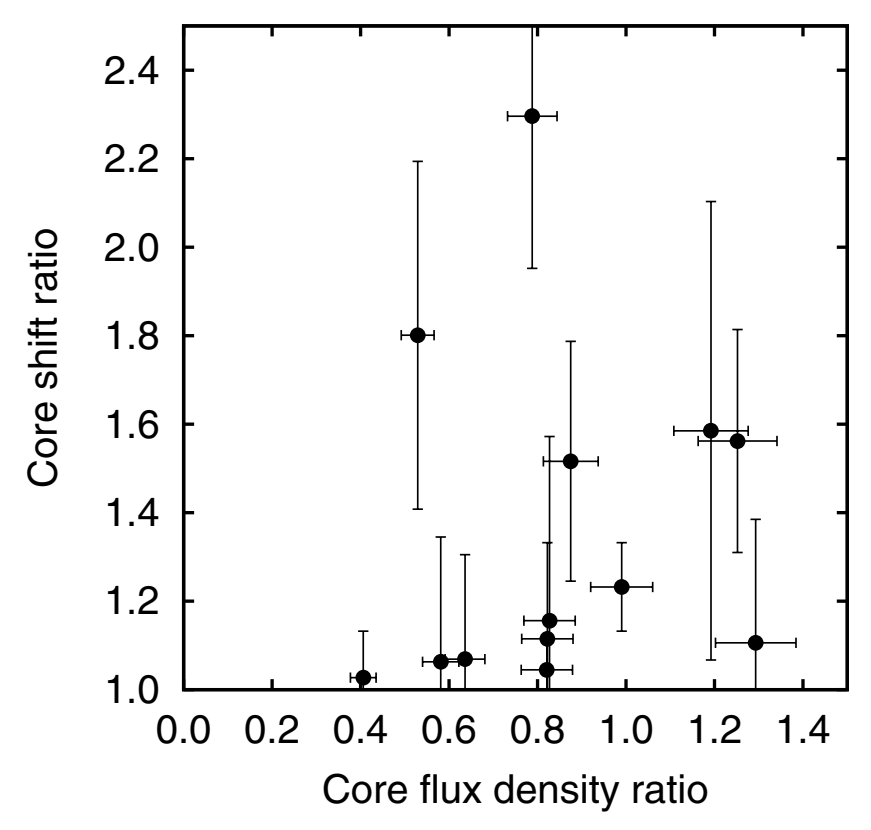

Fig. 6. Ratio of the $X-S$ band core position shift measured at 2002 by Kovalev et al. (2008a) to the one measured in 2007 (this paper) as a function of the $X$ band core flux density ratio at these two epochs. The core shift ratio is defined to be greater than unity, i.e., for each pair of measurements, the higher value of the shift is in the numerator of the ratio. The one source (not shown in this plot), which exhibited a large core shift change without a major change in flux density is W Com $(1219+285)$; it has a core shift ratio of $3.67 \pm 0.36$ and core flux ratio of $1.19 \pm 0.08$.

According to formula (1) in Kovalev et al. (2008a), if jet orientation and velocity remain unchanged, an increasing value of core shift (due to an increase in particle density and/or jet magnetic field strength) should be associated with an increase in the flux density of the core. Figure 6 shows the core shift ratio versus $X$ band core flux density ratio at two epochs (2002 and 2007). No significant correlation is found between the core shift and flux ratios at different epochs. This may reflect that there is no single dominant physical reason for core flares and subsequent core shift variations, despite the suggestion by Kovalev et al. (2008a). A higher accuracy of core-shift ratio measurements is required to probe this in detail.

\section{Summary}

Dedicated VLBA observations of twenty AGN jets showing large frequency-dependent core shifts were conducted at nine frequencies in the $1.4-15.4 \mathrm{GHz}$ range. Significant core shifts have been detected and confirmed in all observed sources. The effect is more pronounced at lower frequencies. The median value of the core shift in the observed sources is 1.21 mas if measured between 1.4 and $15.4 \mathrm{GHz}$ and 0.24 mas between 5.0 and $15.4 \mathrm{GHz}$. The core position, $r_{\mathrm{c}}$, shift as a function of frequency, $v$, is consistent with the $r_{\mathrm{c}}(v) \propto v^{-1}$ pattern expected for a purely synchrotron self-absorbed conical jet in equipartition (Blandford \& Königl 1979; Lobanov 1998). The mean value of the coefficient $k$ in the $r_{\mathrm{c}}(v) \propto v^{-1 / k}$ relation was found to be $k=0.99 \pm 0.14$, while the median is 0.82 . These results support the interpretation of the parsec-scale core as a continuous Blandford-Königl type jet with smooth gradients of physical properties (including opacity) along it. No systematic change with frequency of the power law index in the $r_{\mathrm{c}}(v)$ relation has been convincingly detected. However, some local changes might be present in a few sources, especially at higher frequencies.

The general applicability of the $r_{\mathrm{c}}(v) \propto v^{-1}$ relation to all the observed sources is a promising indication that, if a radio source is strongly core-dominated, its positions obtained using group delays (such as those currently used to define the ICRF2) may be compared to optical counterpart positions directly, with no need to apply a correction for the source core shift (Porcas 2009). This may be important in the era of the future spacebased optical astrometry missions aiming for $\mu$ as-level accuracy. A correction for the core shift in the reference source(s) is 
still required if a high-precision absolute position of a celestial radio source (or tracked spacecraft) is to be determined through a phase-referencing VLBI experiment.

While in this paper we have tried to concentrate on the observational results, independent of specific assumptions about jet geometry and Lorentz factor, in future work based on the dataset presented here we plan to estimate geometry, magnetic field strength, and total (kinetic plus magnetic field) power of the flows and to relate the observed shifts to the properties of the central black hole and broad-line region in its vicinity. Polarization information will be used to constrain the physical interpretation of core shift measurements and to investigate the effect of the core shift on Faraday rotation measurements.

It would be important to confirm and investigate possible changes in the coefficient $k$ in the $r_{\mathrm{c}}(v) \propto v^{-1 / k}$ relation with frequency, which may be hinted at in our observations. To achieve greater accuracy in measuring $k$ one would need to obtain more independent core position measurements across the whole frequency range accessible to VLBI. The use of phase-referenced observations may also be helpful for minimizing ambiguities inevitably associated with the model fitting procedure and quantifying position-measurement errors more accurately.

Calibrated $u v$-data and models described in this paper are available from the first author (KVS) by request.

Acknowledgements. We thank Richard Porcas and the anonymous referee for valuable comments that helped to improve the manuscript. K.V.S. is supported by the International Max-Planck Research School (IMPRS) for Astronomy and Astrophysics at the universities of Bonn and Cologne. Y.Y.K. was supported in part by the return fellowship of the Alexander von Humboldt foundation and the Russian Foundation for Basic Research (RFBR) grant 08-02-00545 and 11-0200368. This work is based on data obtained from the National Radio Astronomy Observatory's Very Long Baseline Array (VLBA), project BK 134. The National Radio Astronomy Observatory is a facility of the National Science Foundation operated under cooperative agreement by Associated Universities, Inc. This research has made use of the NASA/IPAC Extragalactic Database (NED), which is operated by the Jet Propulsion Laboratory, California Institute of Technology, under contract with the National Aeronautics and Space Administration. This research has made use of NASA's Astrophysics Data System.

\section{References}

Afanasiev, V. L., Dodonov, S. N., Moiseev, A. V., et al. 2003, ARep, 47, 458 Aller, H. D., Aller, M. F., Latimer, G. E., \& Hodge, P. E. 1985, ApJS, 59, 513 Aller, M. F., Aller, H. D., \& Hughes, P. A. 1992, ApJ, 399, 16

Aller, M. F., Aller, H. D., \& Hughes, P. A. 2003, Radio Astronomy at the Fringe, ed. J. A. Zensus, M. H. Cohen, \& E. Ros (San Francisco: ASP), in ASP Conf. Ser., 300, 159

Bach, U., Villata, M., Raiteri, C. M., et al. 2006, A\&A, 456, 105

Beasley, A. J., Gordon, D., Peck, A. B., et al. 2002, ApJS, 141, 13

Biretta, J. A., Moore, R. L., \& Cohen, M. H. 1986, ApJ, 308, 93

Blandford, R. D., \& Königl, A. 1979, ApJ, 232, 34

Croke, S. M., O’Sullivan, S. P., \& Gabuzda, D. C. 2010, MNRAS, 402, 259

Fey, A. L., \& Charlot, P. 1997, ApJS, 111, 95

Fey, A. L., Ma, C., Arias, E. F., et al. 2004, AJ, 127, 3587

Fey, A. L., Gordon, D., \& Jacobs, C. S. 2010, IERS/IVS Working Group, The

Second Realization of the International Celestial Reference Frame by Very Long Baseline Interferometry, IERS Technical Note No. 35

Finke, J. D., Shields, J. C., Böttcher, M., \& Basu, S. 2008, A\&A, 477, 513

Fomalont, E. B. 1999, in Synthesis Imaging in Radio Astronomy II, ed. G. B. Taylor, C. L. Carilli, \& R. A. Perley (San Francisco: ASP), ASP Conf. Ser., 180,301
Fomalont, E. B., Petrov, L., MacMillan, D. S., Gordon, D., \& Ma, C. 2003, AJ, 126,2562

Greisen, E. W. 2003, in Information Handling in Astronomy - Historical Vistas, ed. A. Heck, Astrophys. Space Sci. Lib., 285, 109

Hildebrand, C. E., Iijima, B. A., Kroger, P. M., Folkner, W. M., \& Edwards, C. D. 1994, in The Telecommunications and Data Acquisition Report, ed. J. H. Yuen, 46

Homan, D. C., Ojha, R., Wardle, J. F. C., et al. 2002, ApJ, 568, 99

Högbom, J. A. 1974, A\&AS, 15, 417

Kadler, M., Ros, E., Lobanov, A. P., Falcke, H., \& Zensus, J. A. 2004, A\&A, 426, 481

Komatsu, E., Dunkley, J., Nolta, M. R., et al. 2009, ApJS, 180, 330

Königl, A. 1981, ApJ, 243, 700

Kovalev, Y. Y., Nizhelsky, N. A., Kovalev, Y. A., et al. 1999, A\&AS, 139, 545

Kovalev, Y. Y., Kovalev, Yu. A., Nizhelsky, N. A., \& Bogdantsov, A. B. 2002, PASA, 19, 83

Kovalev Y. Y., Kellermann K. I., Lister M. L., et al. 2005, AJ, 130, 2473

Kovalev, Y. Y., Petrov, L., Fomalont, E. B., \& Gordon, D. 2007, AJ, 133, 1236

Kovalev, Y. Y., Lobanov, A. P., Pushkarev, A. B., \& Zensus, J. A. 2008a, A\&A, 483, 759

Kovalev, Y. Y., Lobanov, A. P., \& Pushkarev, A. B. 2008b, Mem. Soc. Astron. Ital., 79, 1153

Lee, S.-S., Lobanov, A. P., Krichbaum, T. P., et al. 2008, AJ, 136, 159

Lindegren, L. 2007, Highlights of Astronomy, 14, 481

Lobanov, A. P. 1998, A\&A, 330, 79

Lobanov, A. P. 2007, Ap\&SS, 311, 263

Ma, C., Arias, E. F., Eubanks, T. M., et al. 1998, AJ, 116, 516

Marcaide, J. M., \& Shapiro, I. I. 1984, ApJ, 276, 56

Marcaide, J. M., Shapiro, I. I., Corey, B. E., et al. 1985, A\&A, 142, 71

Marscher, A. P. 2006, in Relativistic Jets: The Common Physics of AGN, Microquasars, and Gamma-Ray Bursts, AIP Conf. Proc., 856, 1

Marscher, A. P. 2008, in Extragalactic Jets: Theory and Observation from Radio to Gamma Ray, ed. T. A. Rector, \& D. S. De Young (San Francisco: ASP), ASP Conf. Ser., 386, 437

Napier, P. J. 1994, in Very High Angular Resolution Imaging, ed. J. G. Robertson, \& W. J. Tango, Proc. IAU Symp., 158, 117

O'Sullivan, S. P., \& Gabuzda, D. C. 2009, MNRAS, 400, 26

Perryman, M. A. C., de Boer, K. S., Gilmore, G., et al. 2001, A\&A, 369, 339

Petrov, L., Kovalev, Y. Y., Fomalont, E. B., \& Gordon, D. 2005, AJ, 129, 1163

Petrov, L., Kovalev, Y. Y., Fomalont, E. B., \& Gordon, D. 2006, AJ, 131, 1872

Petrov, L., Kovalev, Y. Y., Fomalont, E. B., \& Gordon, D. 2008, AJ, 136, 580

Petrov, L., Gordon, D., Gipson, J., et al. 2009, J. Geod., 83, 859

Pogrebenko, S. V., Gurvits, L. I., Avruch, I. M., \& Cimo, G. 2009, in Eur. Planet. Sci. Congress, 199

Porcas, R. W. 2009, A\&A, 505, L1

Press, W. H., Teukolsky, S. A., Vetterling, W. T., \& Flannery, B. P. 2002, Numerical Recipes in C, 2nd ed. (CUP)

Rioja, M. J., Marcaide, J. M., Elosegui, P., \& Shapiro, I. I. 1997, A\&A, 325, 383

Rioja, M. J., Dodson, R., Porcas, R. W., Suda, H., \& Colomer, F. 2005, in Proceedings of the 17th Working Meeting on European VLBI for Geodesy and Astrometry, ed. Vennebusch, \& Nothnagel, 125 [arXiv: astro-ph/0505475]

Ros, E., \& Lobanov, A. P. 2002, in Proceedings of the 5th Scientific Meeting of the Spanish Astronomical Society, Highlights of Spanish Astrophysics (III), ed. J. Gallego, J. Zamorano, \& N. Cardiel (Kluwer), Astrophys. Space Sci. Lib. [arXiv: astro-ph/0211200]

Sekido, M., Ichikawa, R., Osaki, H., et al. 2004, in International VLBI Service for Geodesy and Astrometry General Meeting Proceedings, ed. N. R. Vandenberg, \& K. D. Baver, 258

Shepherd, M. C. 1997, in Astronomical Data Analysis Software and Systems VI, ed. G. Hunt, \& H. E. Payne (San Francisco: ASP), ASP Conf. Ser., 125, 77

Unwin, S. C., Wehrle, A. E., Urry, C. M., et al. 1994, ApJ, 432, 103

Véron-Cetty, M.-P., \& Véron, P. 2010, A\&A, 518, A10

Walker, R. C., Dhawan, V., Romney, J. D., Kellermann, K. I., \& Vermeulen, R. C. 2000, ApJ, 530, 233

Yang, J., Gurvits, L. I., Frey, S., \& Lobanov, A. P. 2008, in Proc. 10th AsianPacific Regional IAU Meeting, ed. S. N. Zhang, Y. Li, \& Q. J. Yu (National Observatories of China Press) [arXiv : 0811.2926]

Zensus, J. A., Cohen, M. H., \& Unwin, S. C. 1995, ApJ, 443, 35

Pages 12 to 31 are available in the electronic edition of the journal at http://www . aanda. org 

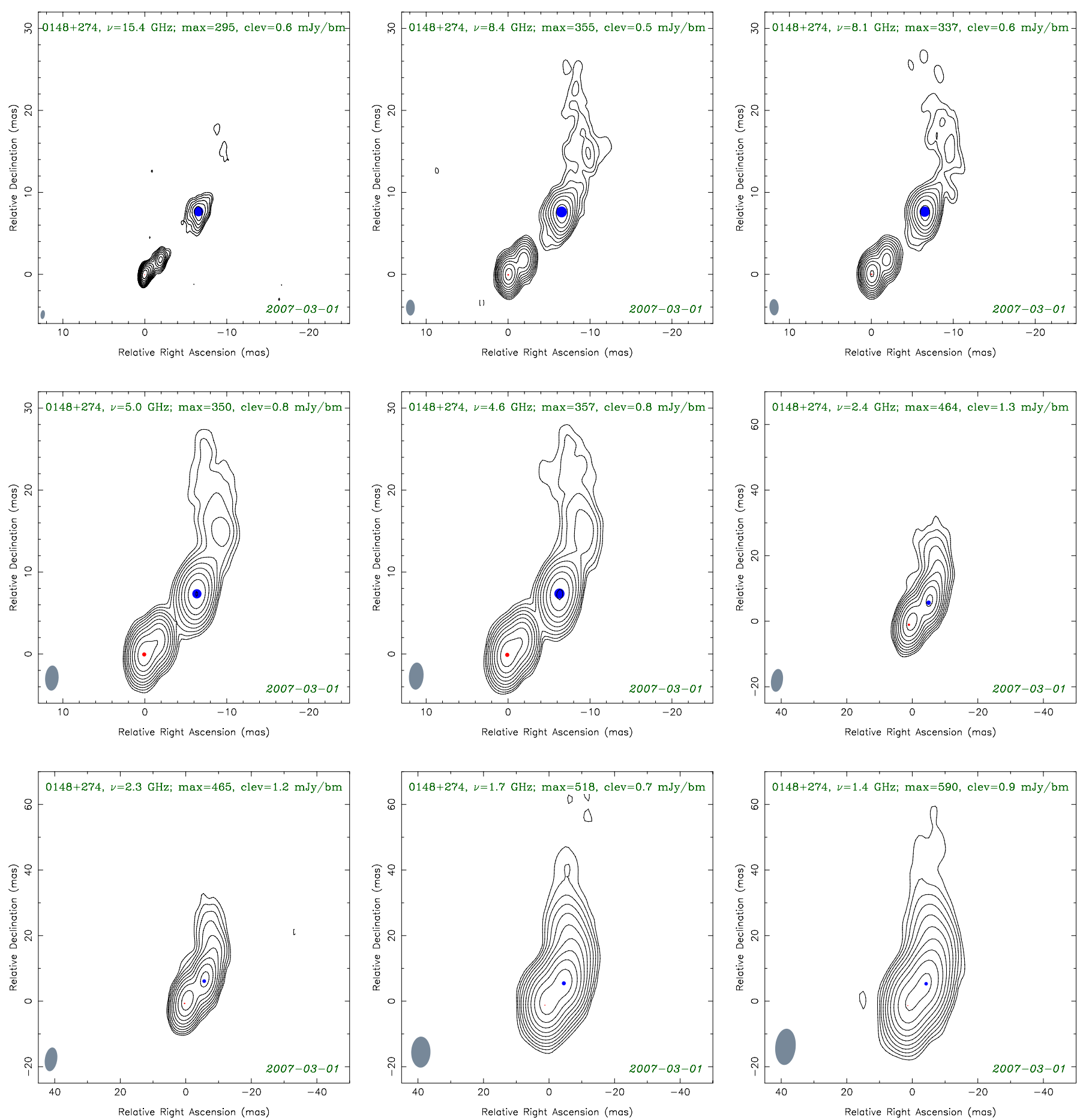

Fig. 1. Naturally weighted CLEAN images of the observed sources between 1.4 and $15 \mathrm{GHz}$. The lowest contour value "clev" is chosen at four times the rms noise, the peak brightness is given by "max". The contour levels increase by factors of two. The dashed contours indicate negative brightness. The beam's full width at half maximum (FWHM) is shown in the bottom left corner of the images in gray. An epoch of observation is shown in the bottom right corner. Red and blue spots indicate the positions and sizes (FWHM) of Gaussian model components for the core and the jet features, respectively. 
K. V. Sokolovsky et al.: A VLBA survey of the core shift effect in AGN jets. I.
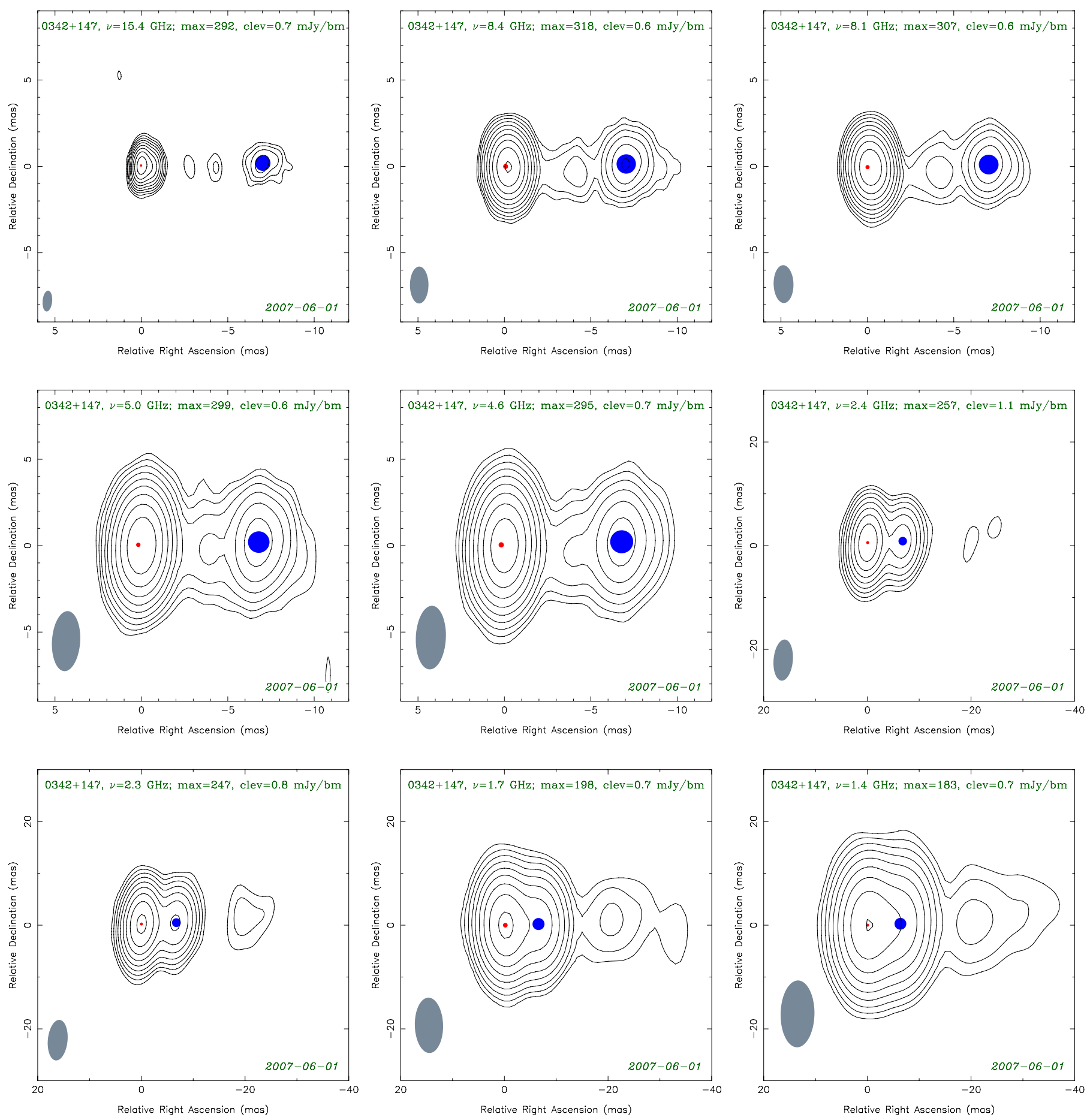

Fig. 1. continued. 
A\&A 532, A38 (2011)
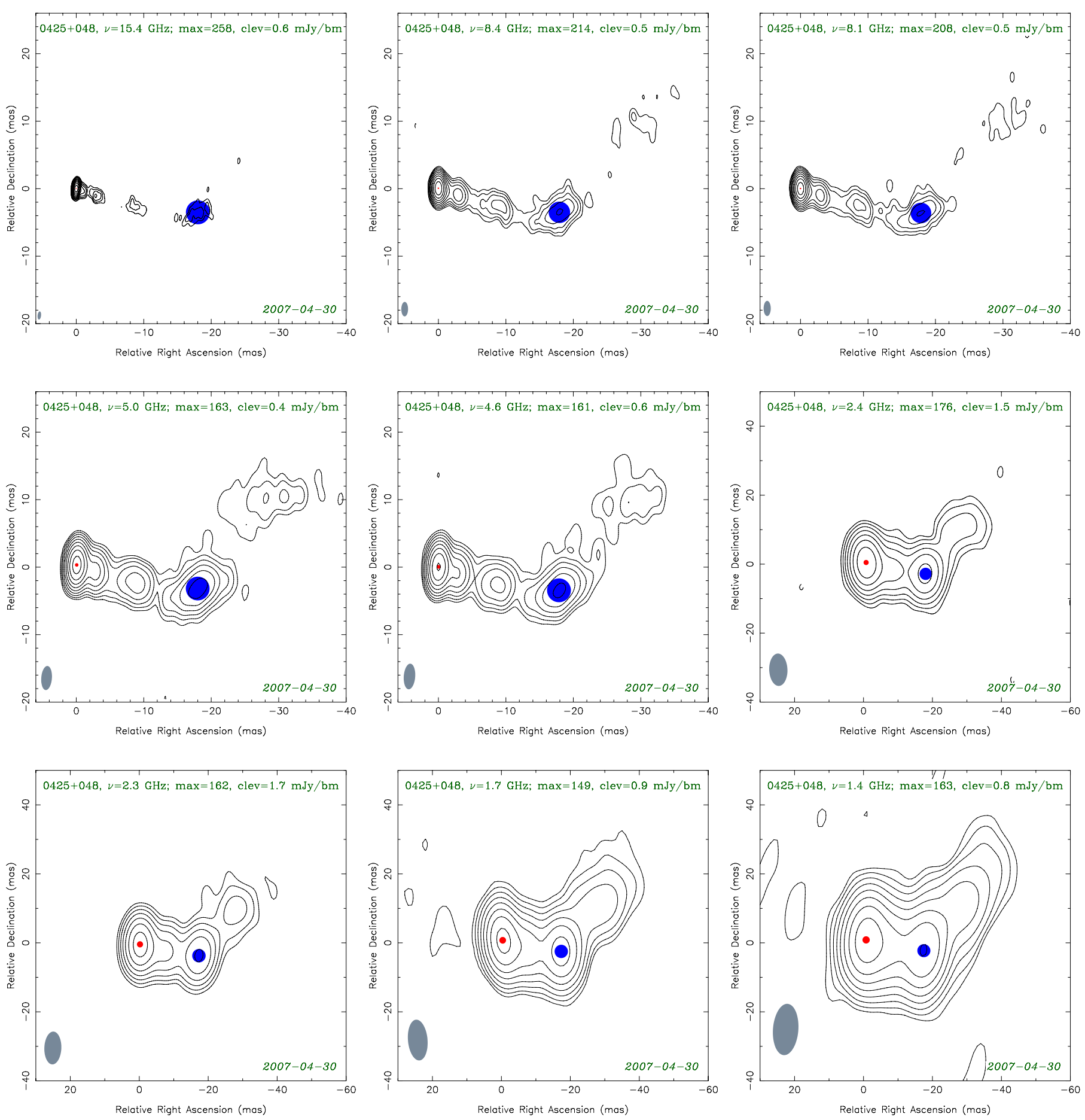

Fig. 1. continued. 
K. V. Sokolovsky et al.: A VLBA survey of the core shift effect in AGN jets. I.
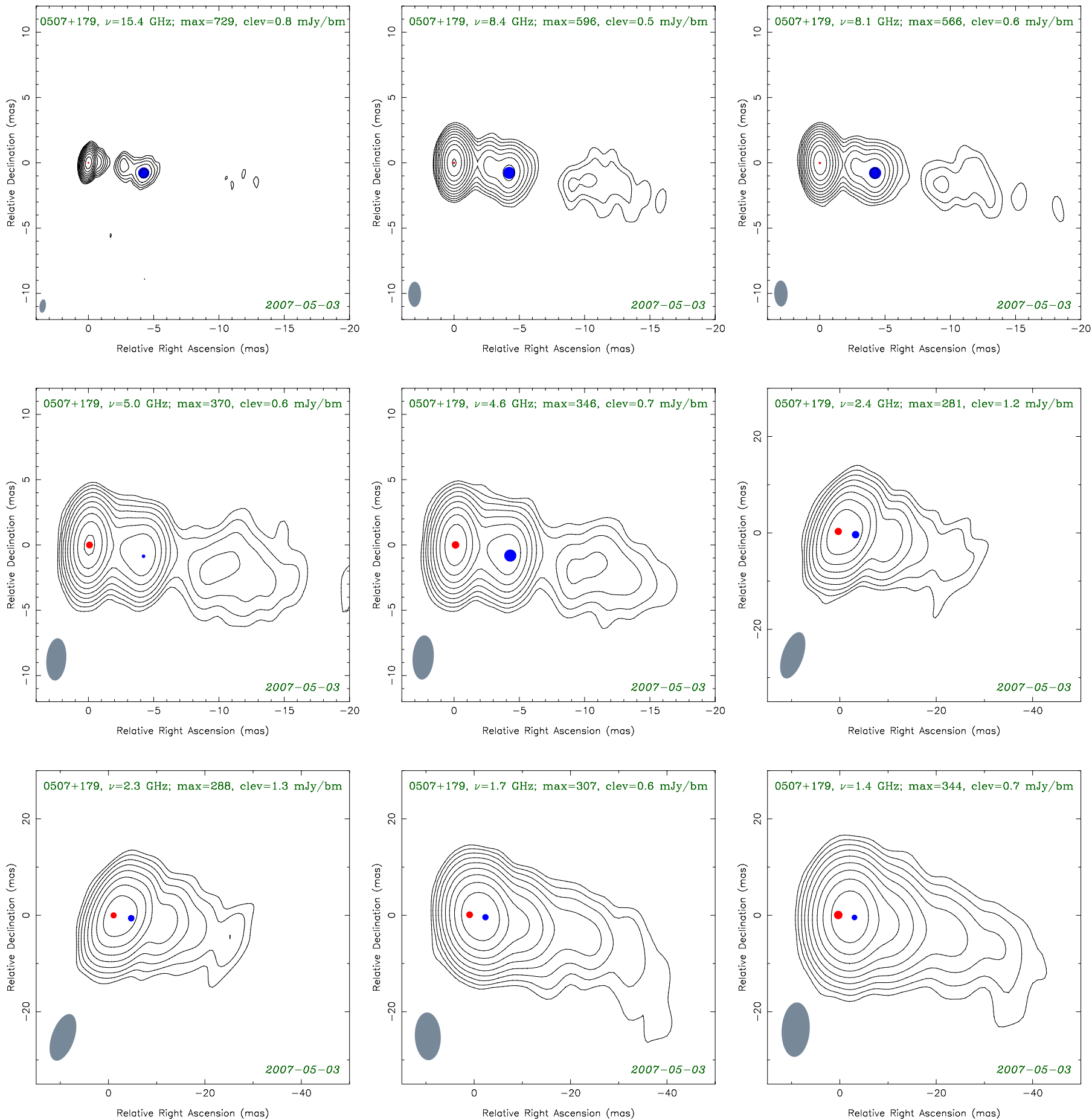

Fig. 1. continued. 
A\&A 532, A38 (2011)
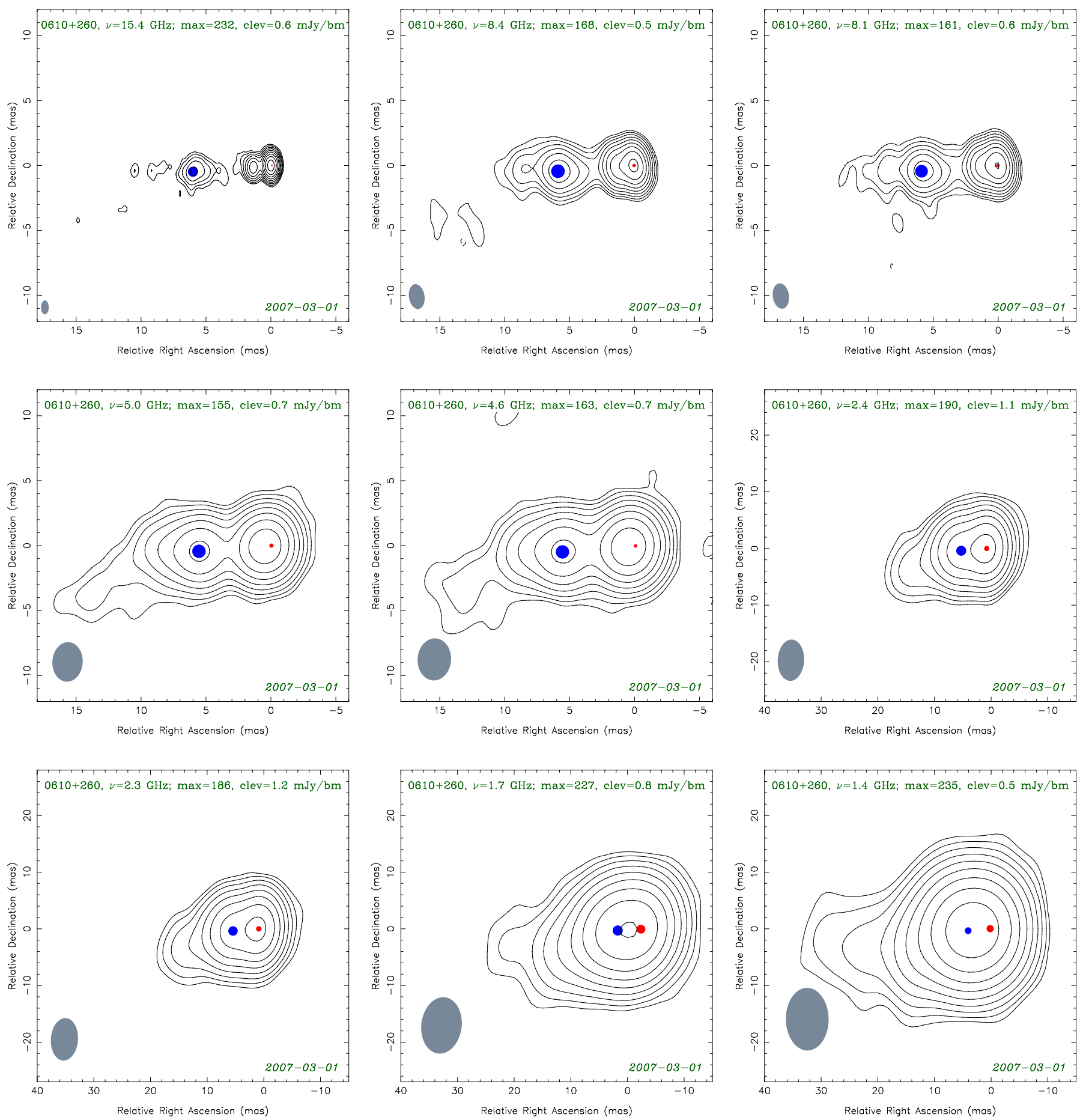

Fig. 1. continued. 
K. V. Sokolovsky et al.: A VLBA survey of the core shift effect in AGN jets. I.
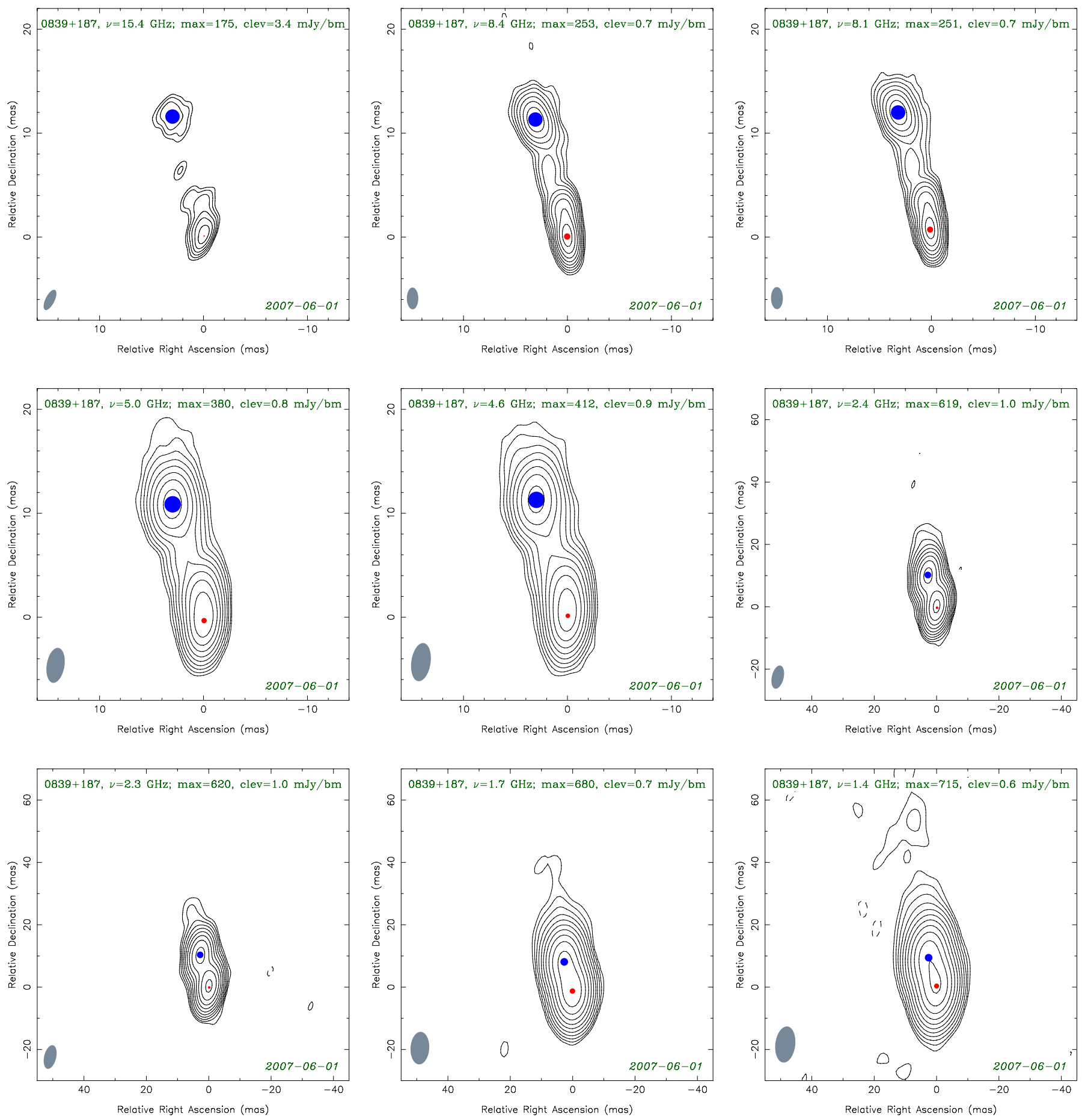

Fig. 1. continued. 
A\&A 532, A38 (2011)
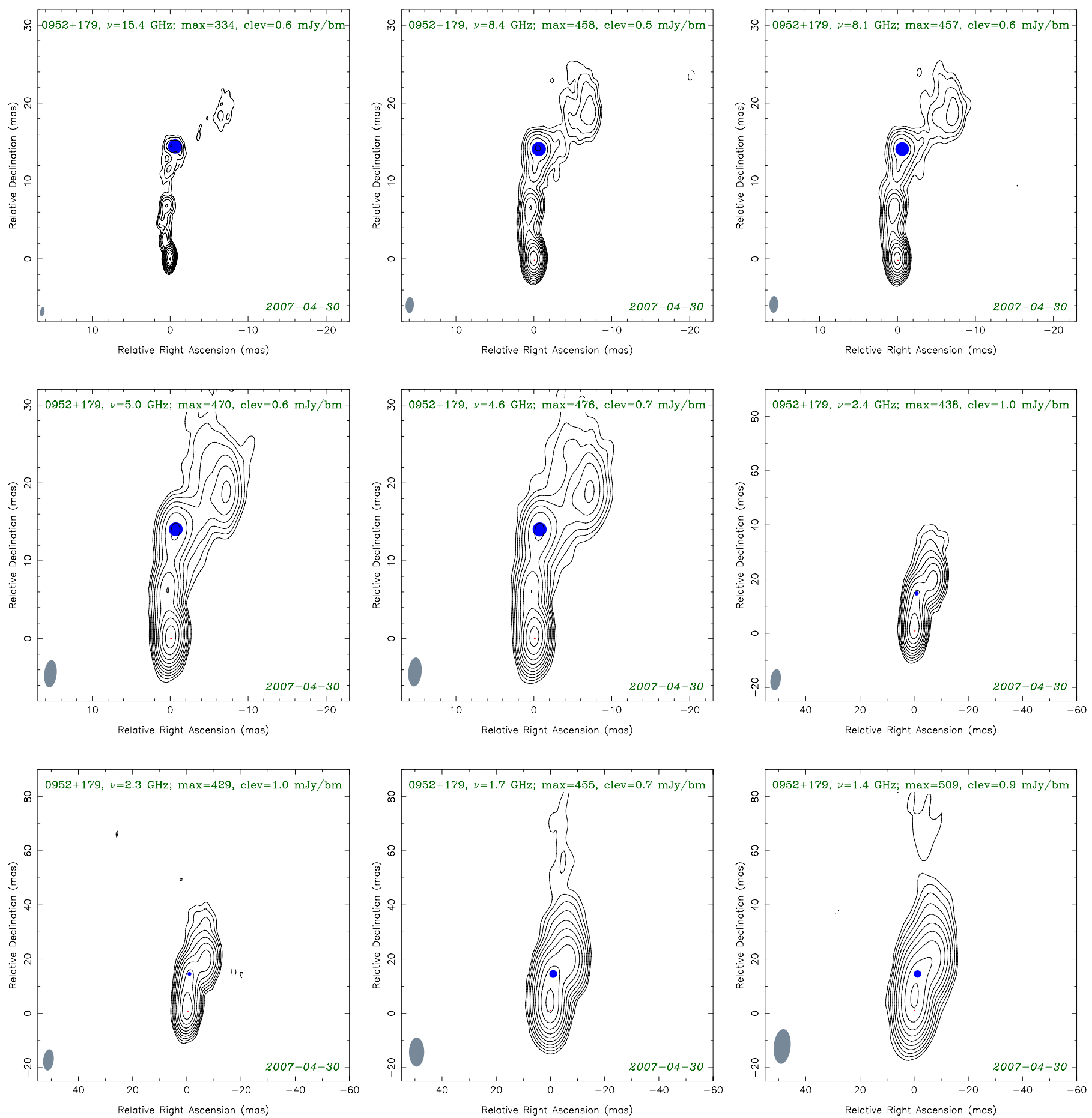

Fig. 1. continued. 
K. V. Sokolovsky et al.: A VLBA survey of the core shift effect in AGN jets. I.
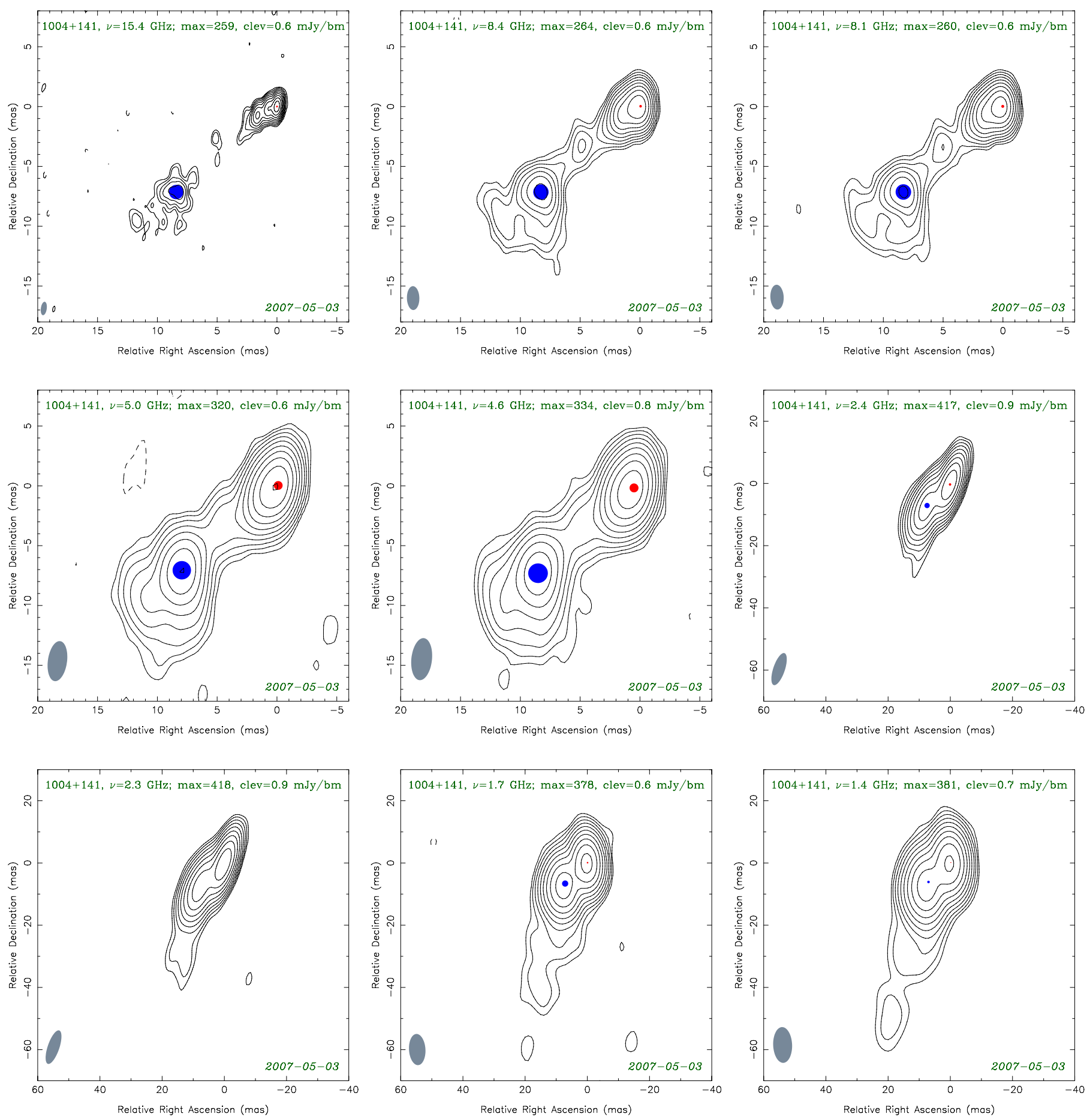

Fig. 1. continued. 
A\&A 532, A38 (2011)
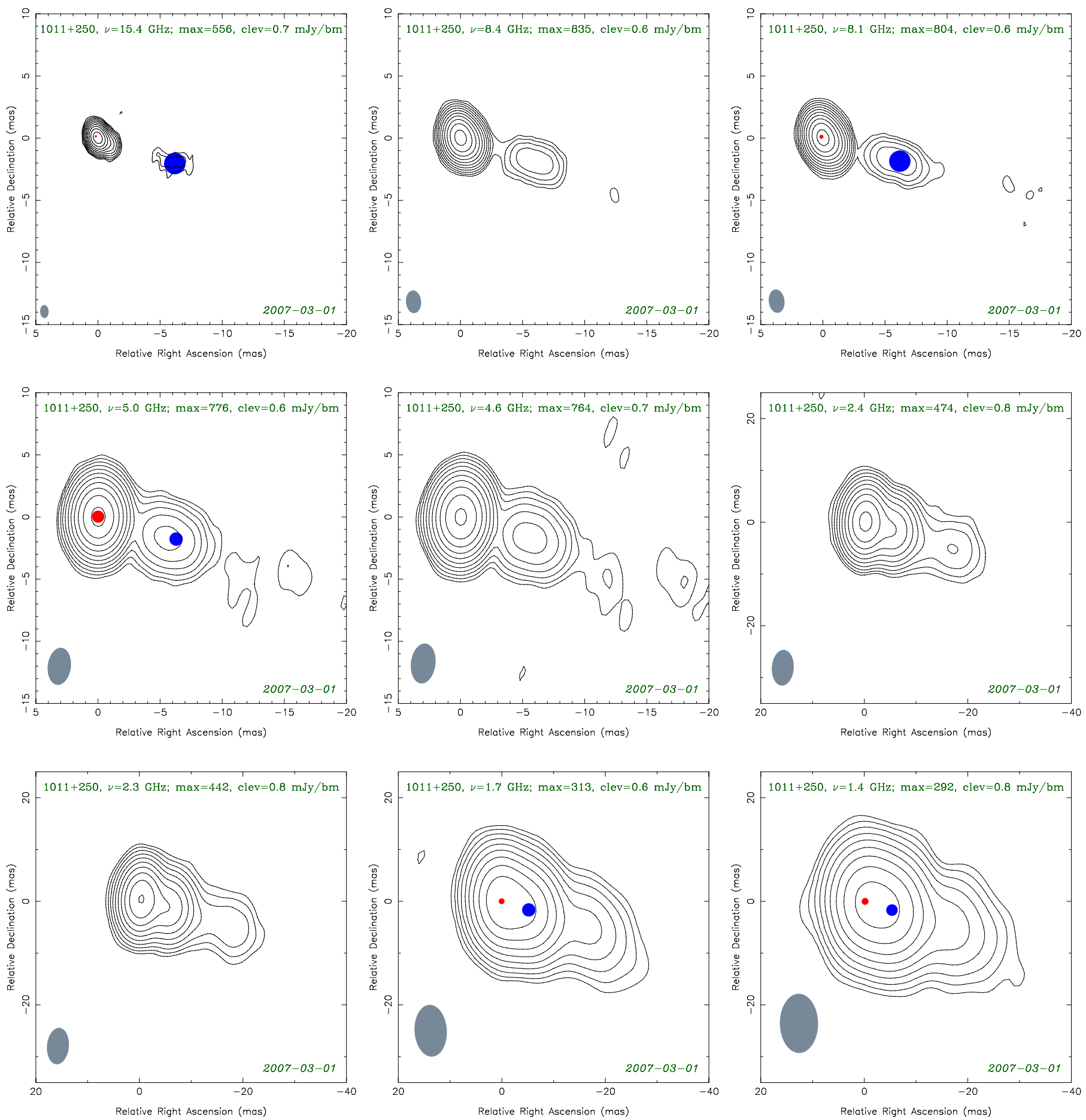

Fig. 1. continued. 
K. V. Sokolovsky et al.: A VLBA survey of the core shift effect in AGN jets. I.
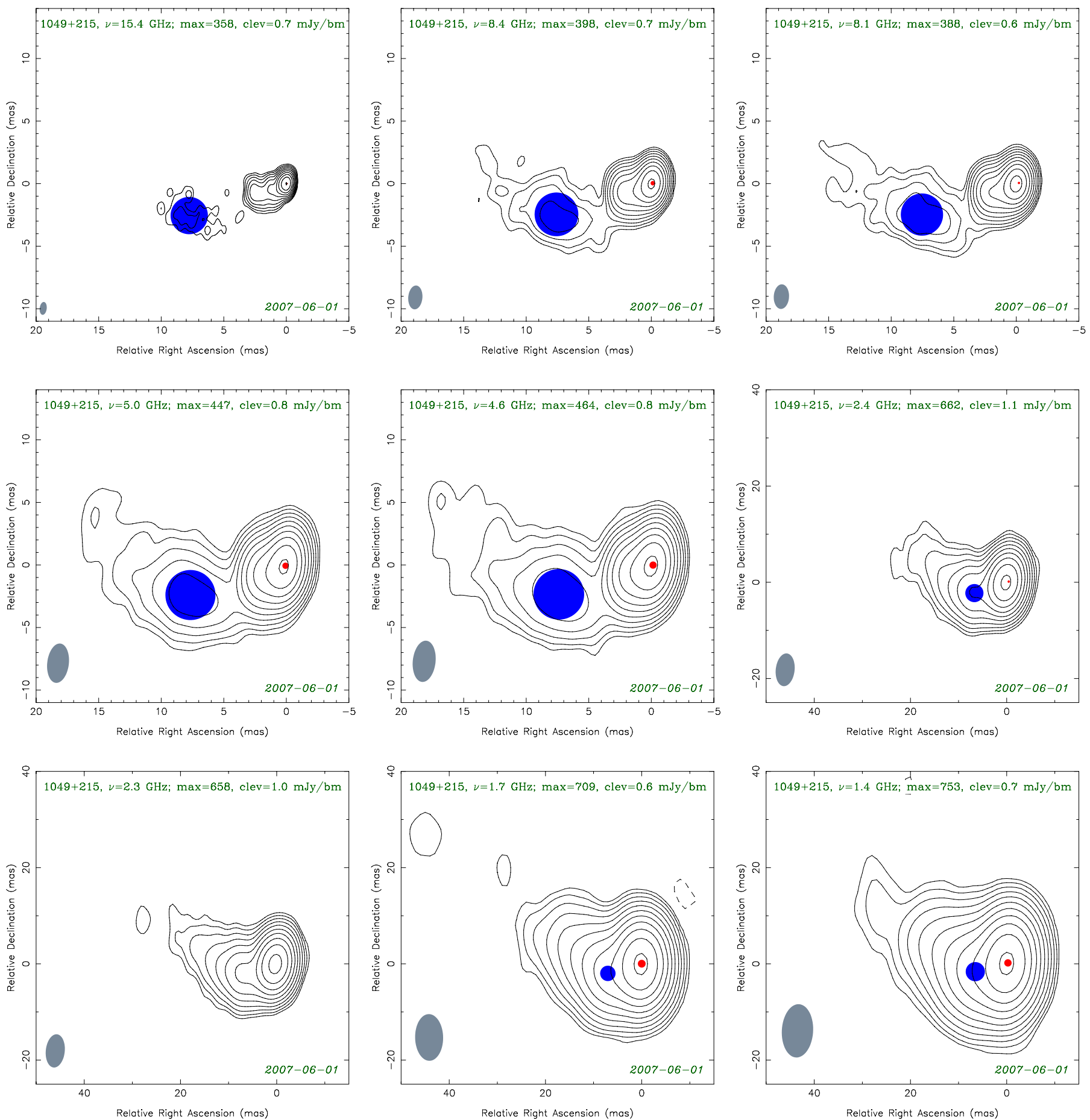

Fig. 1. continued. 
A\&A 532, A38 (2011)
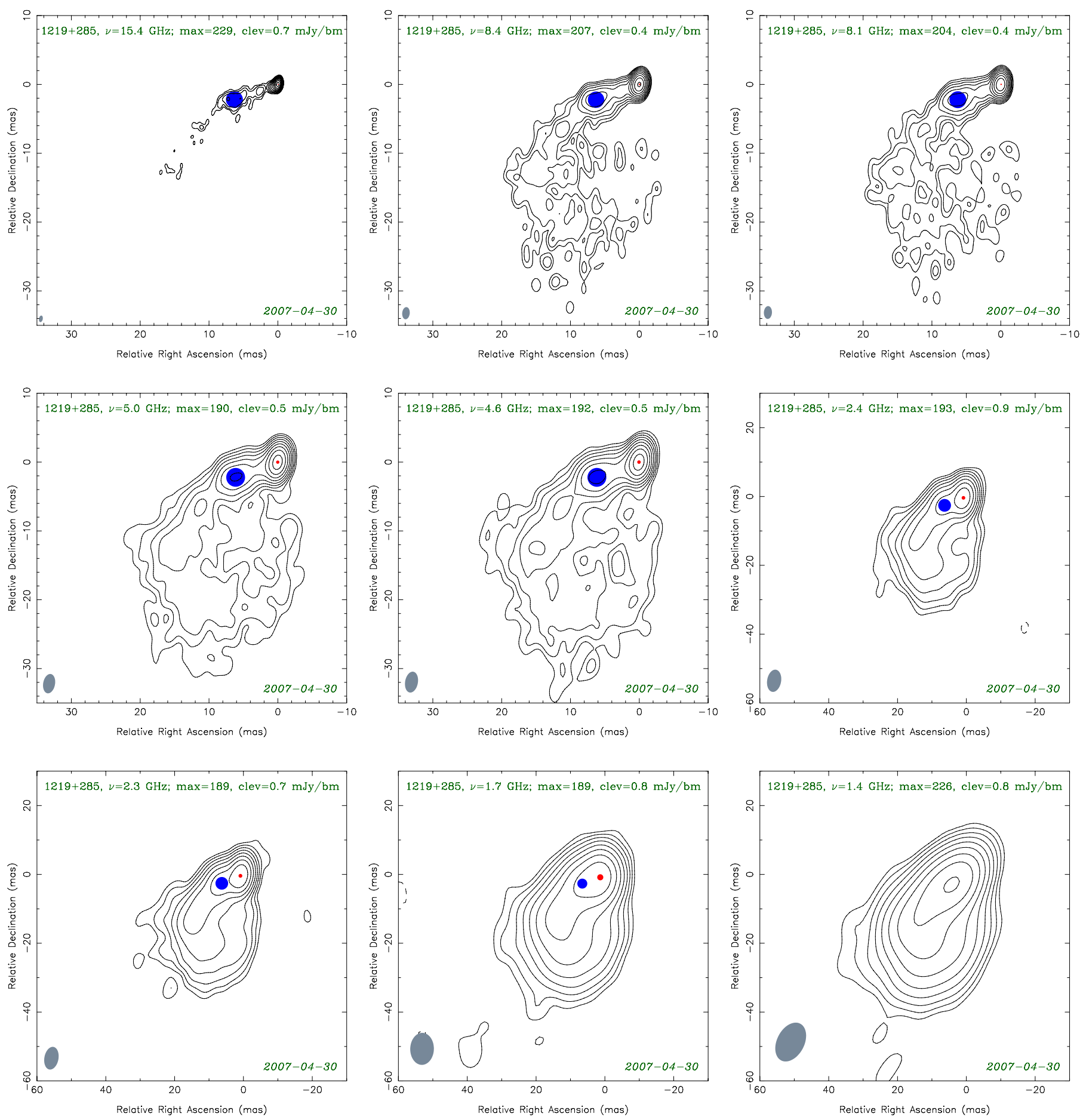

Fig. 1. continued. 
K. V. Sokolovsky et al.: A VLBA survey of the core shift effect in AGN jets. I.
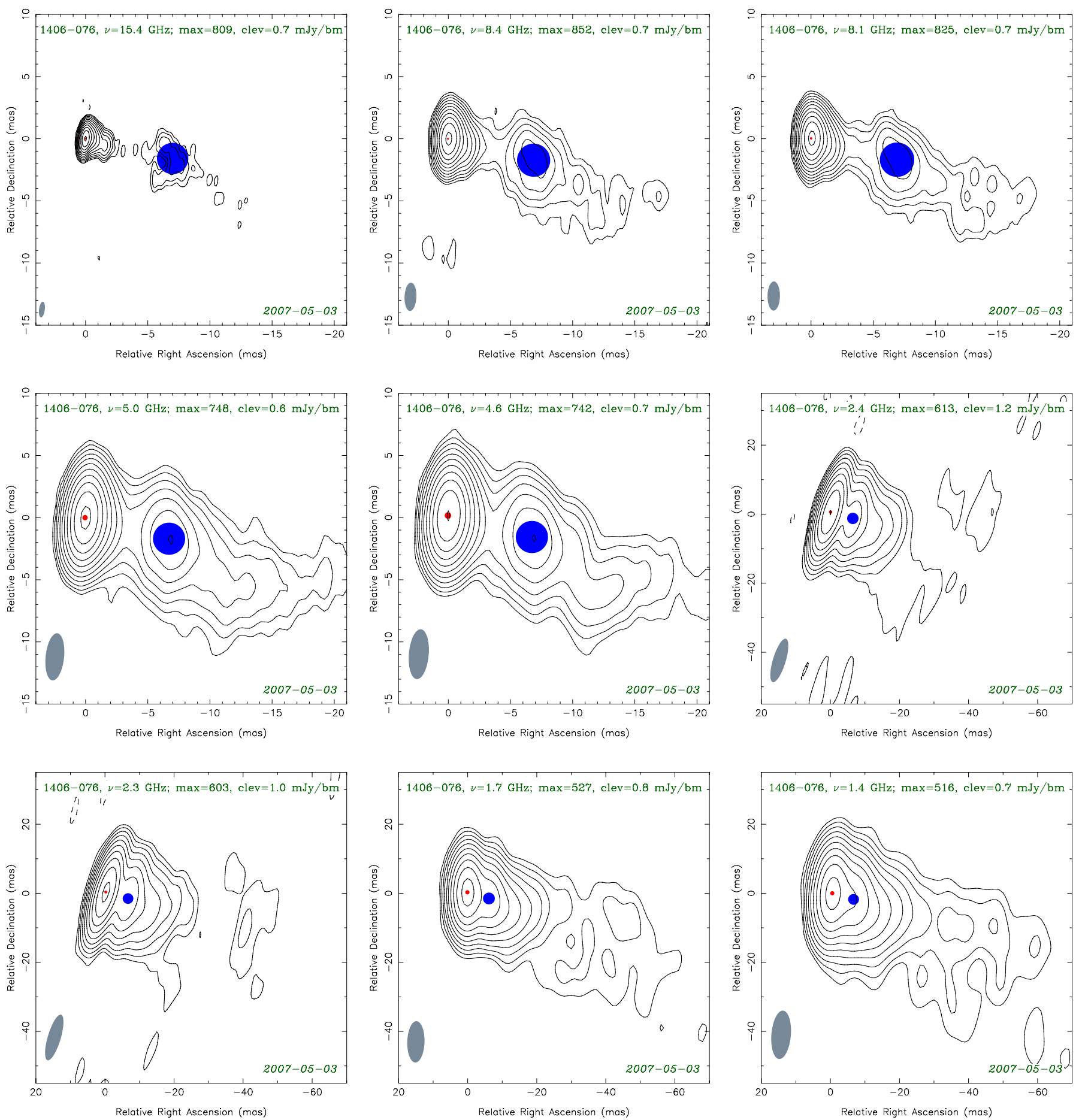

Fig. 1. continued. 
A\&A 532, A38 (2011)
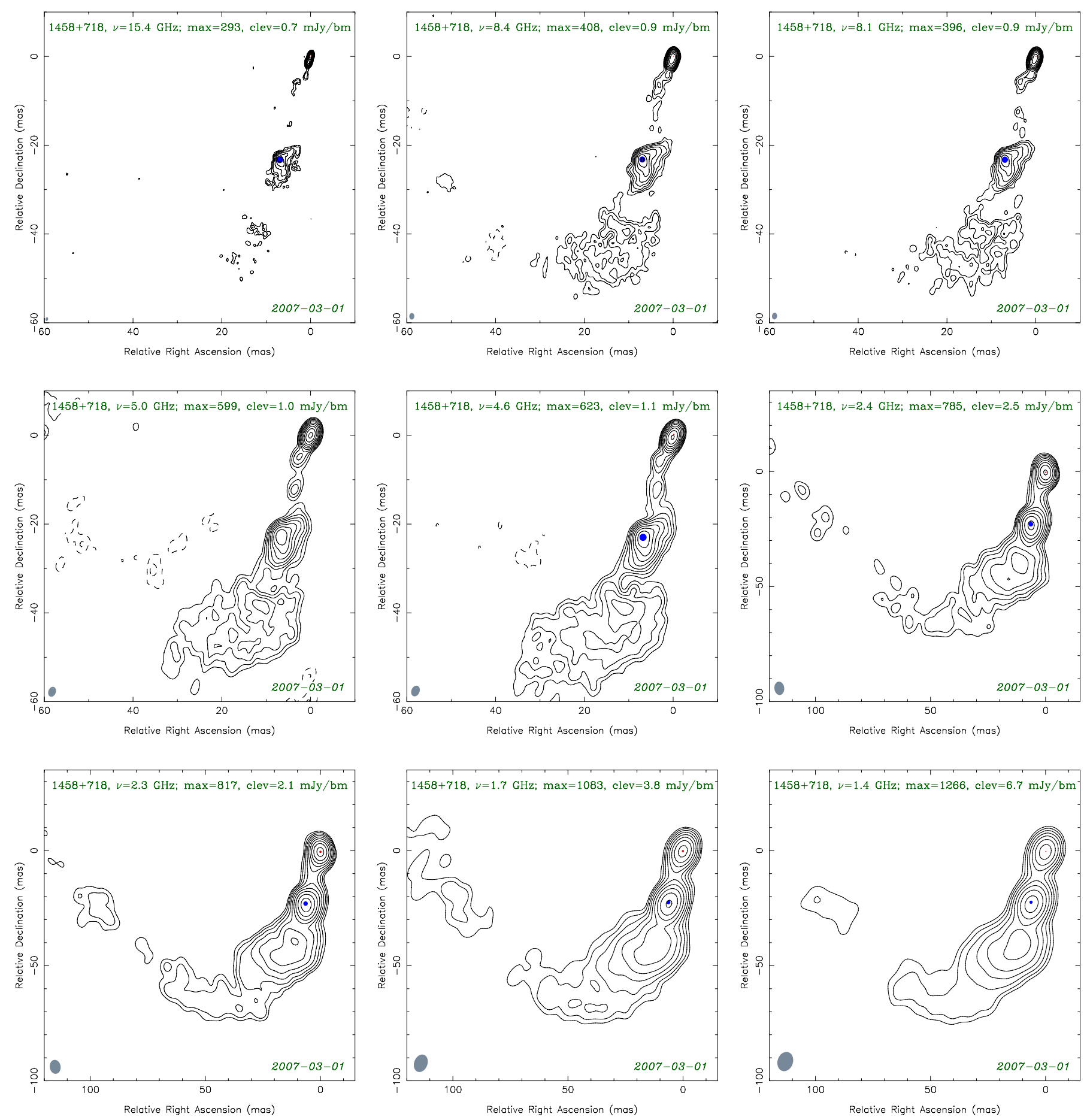

Fig. 1. continued. 
K. V. Sokolovsky et al.: A VLBA survey of the core shift effect in AGN jets. I.
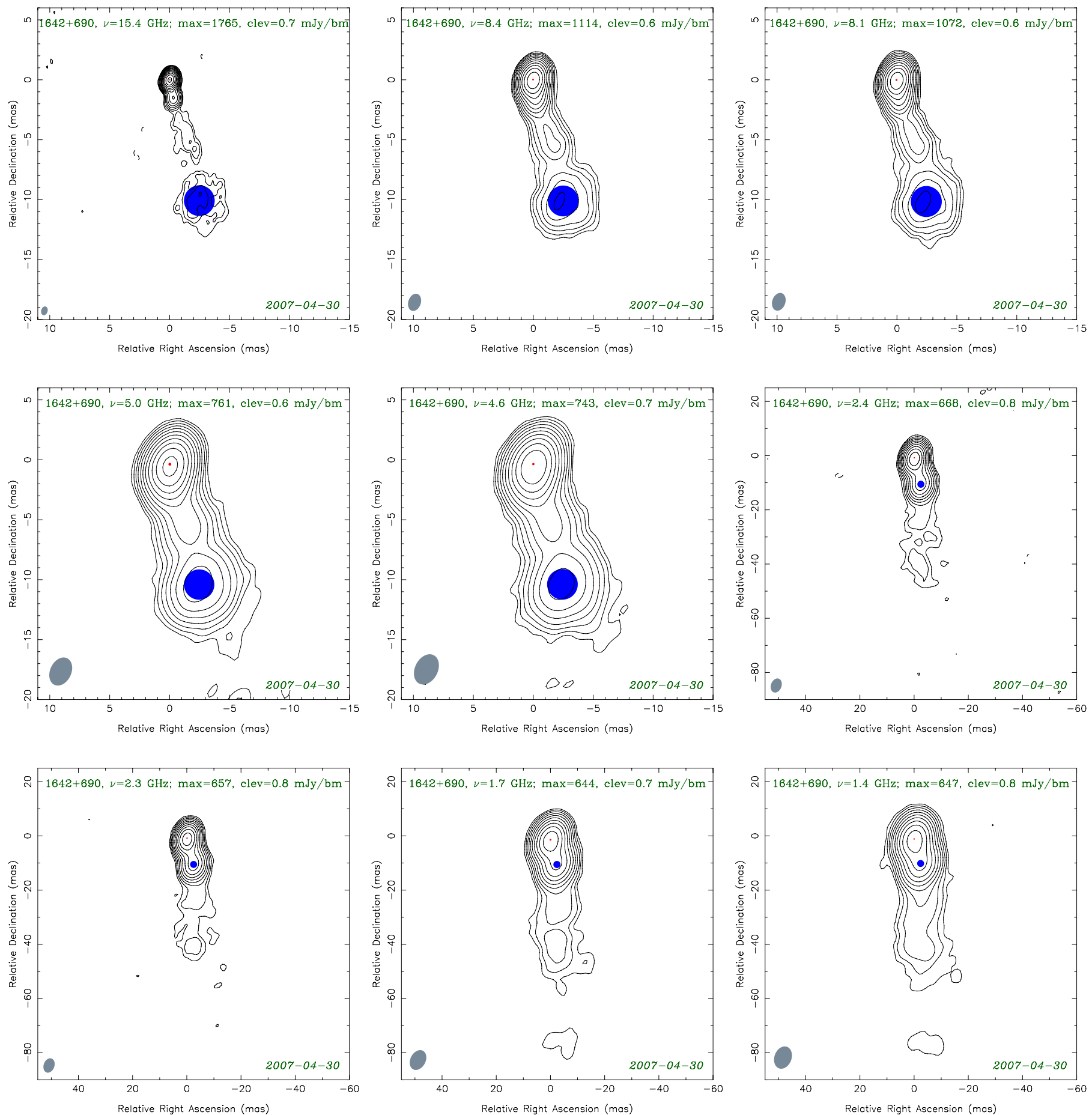

Fig. 1. continued. 
A\&A 532, A38 (2011)
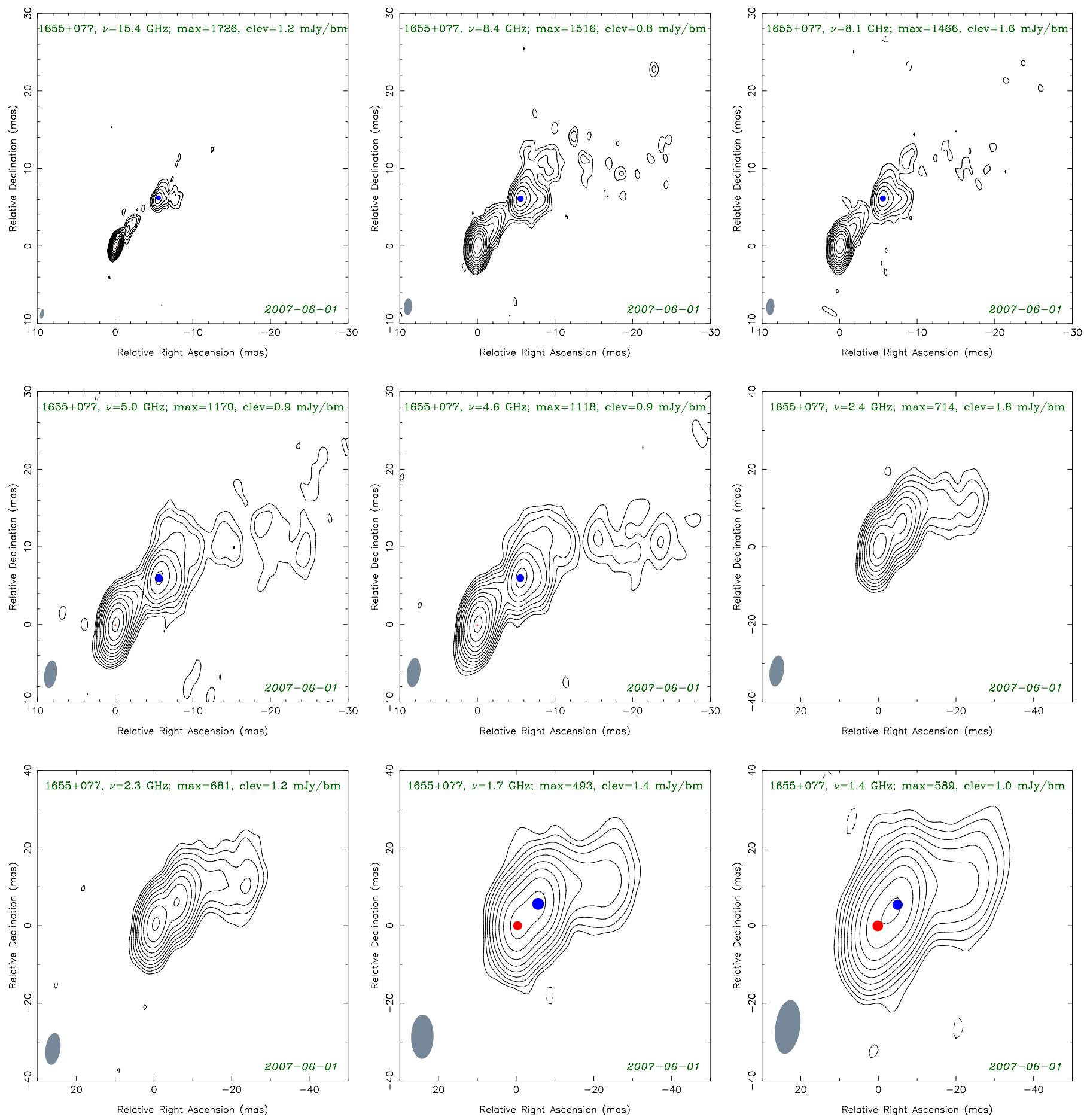

Fig. 1. continued. 
K. V. Sokolovsky et al.: A VLBA survey of the core shift effect in AGN jets. I.
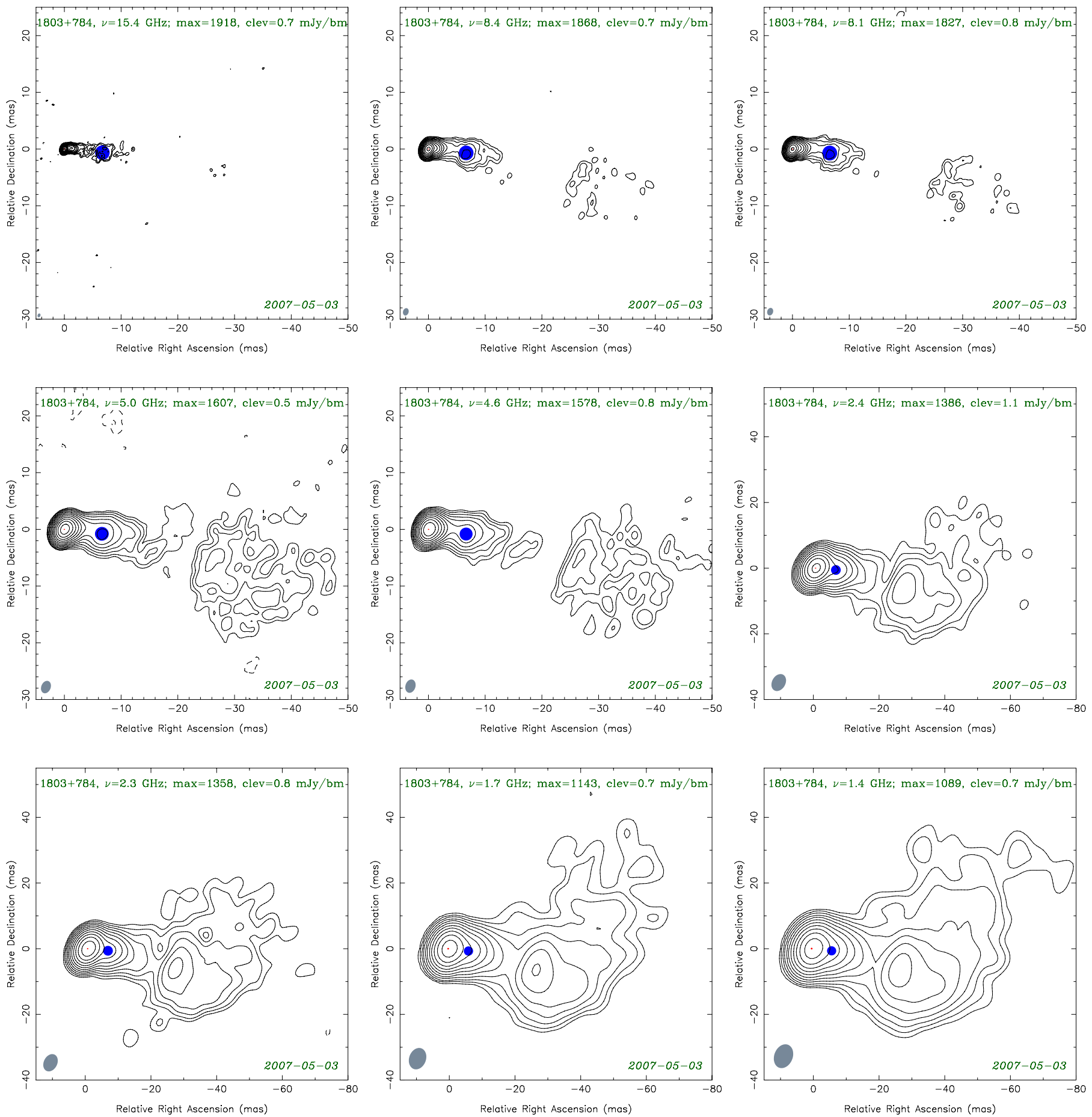

Fig. 1. continued. 
A\&A 532, A38 (2011)
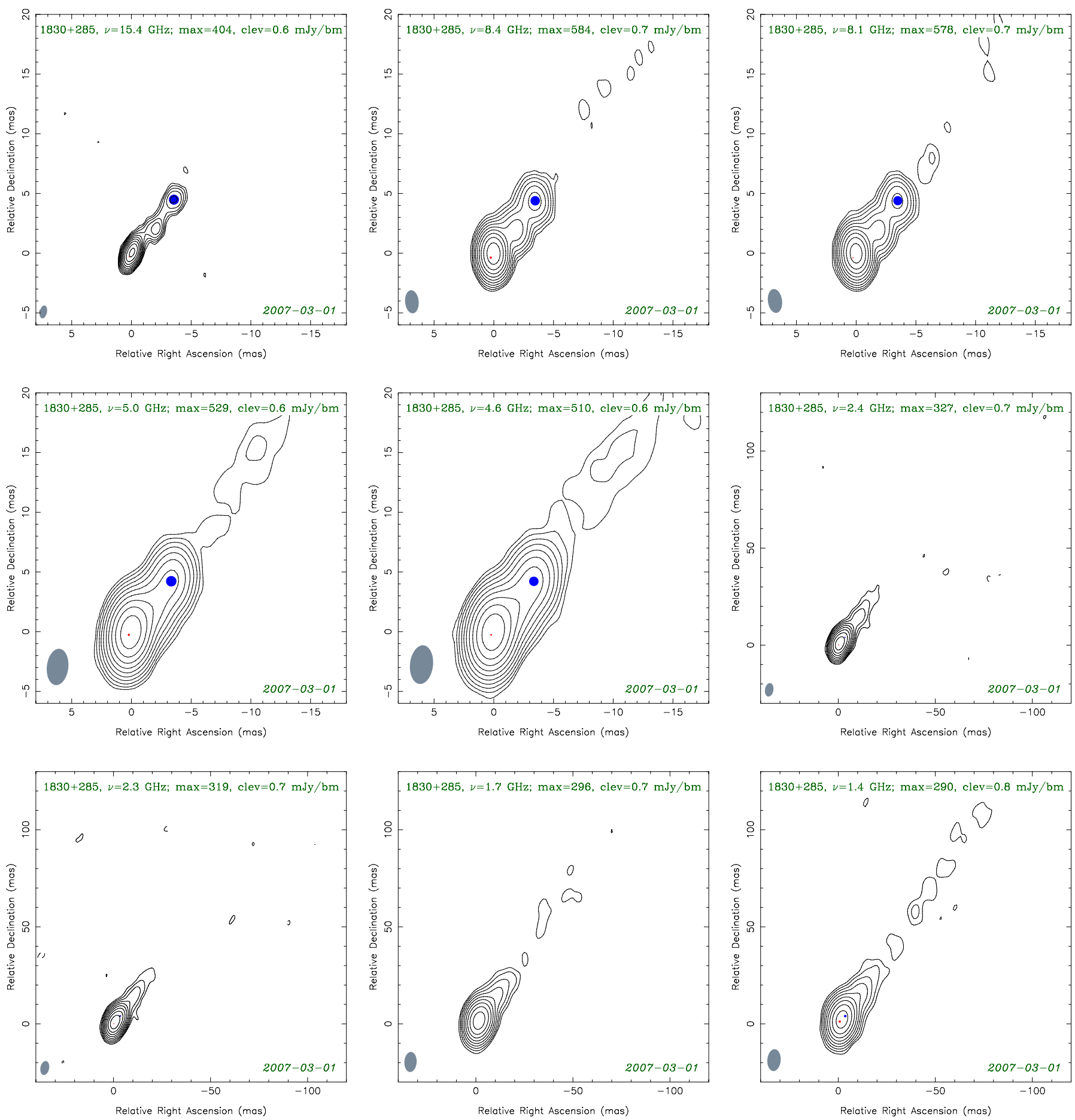

Fig. 1. continued. 
K. V. Sokolovsky et al.: A VLBA survey of the core shift effect in AGN jets. I.
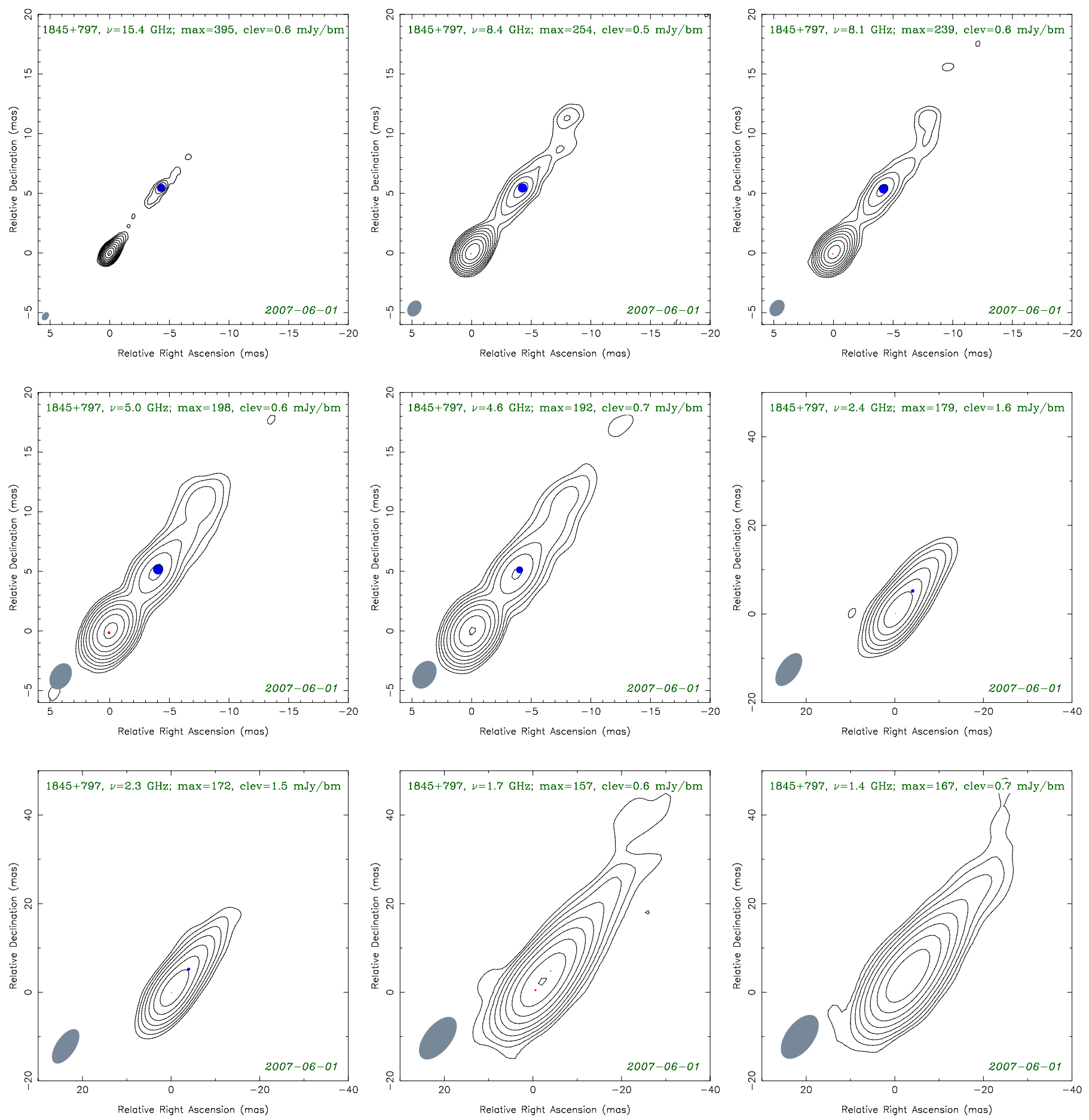

Fig. 1. continued. 
A\&A 532, A38 (2011)
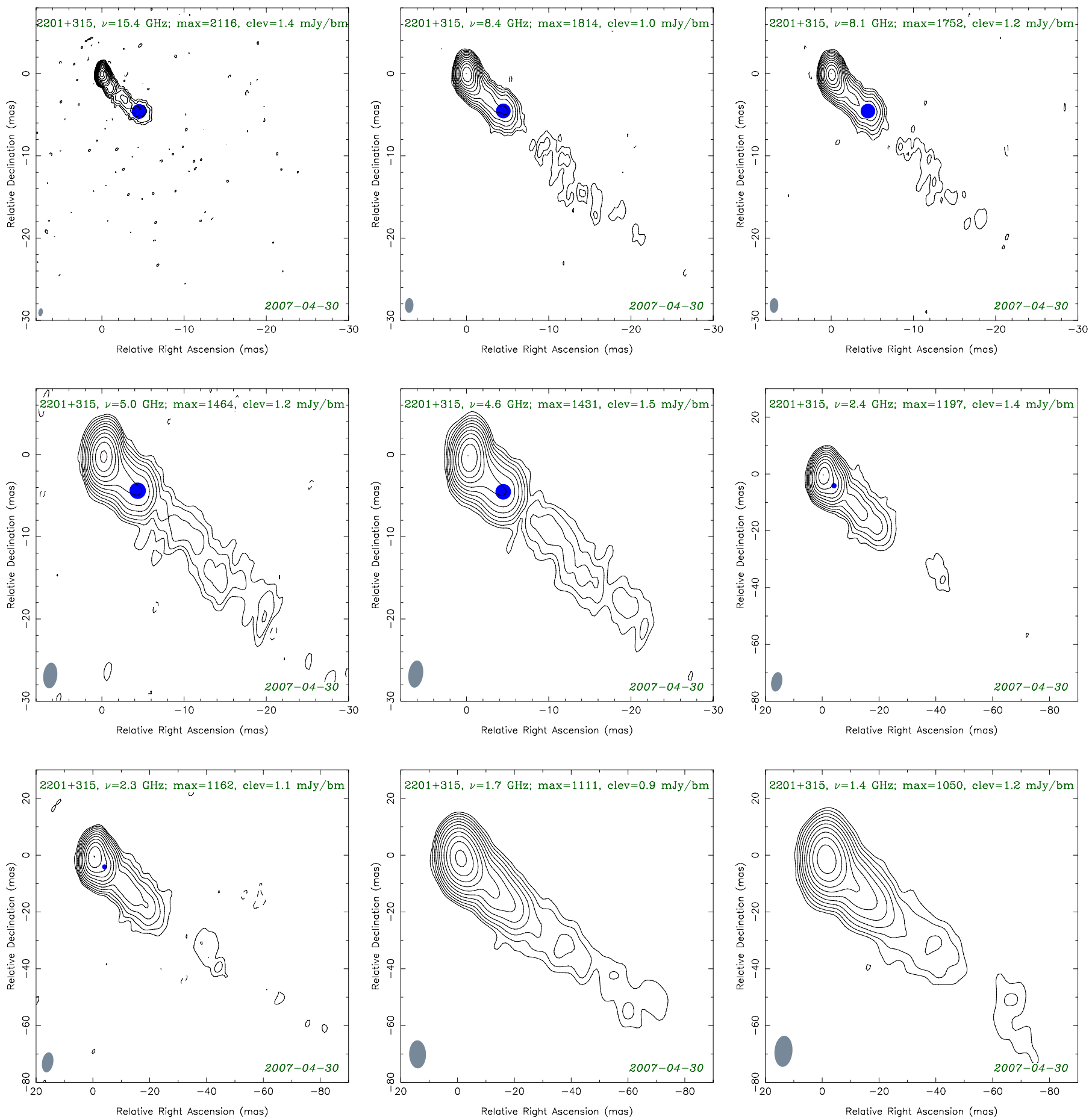

Fig. 1. continued. 
K. V. Sokolovsky et al.: A VLBA survey of the core shift effect in AGN jets. I.
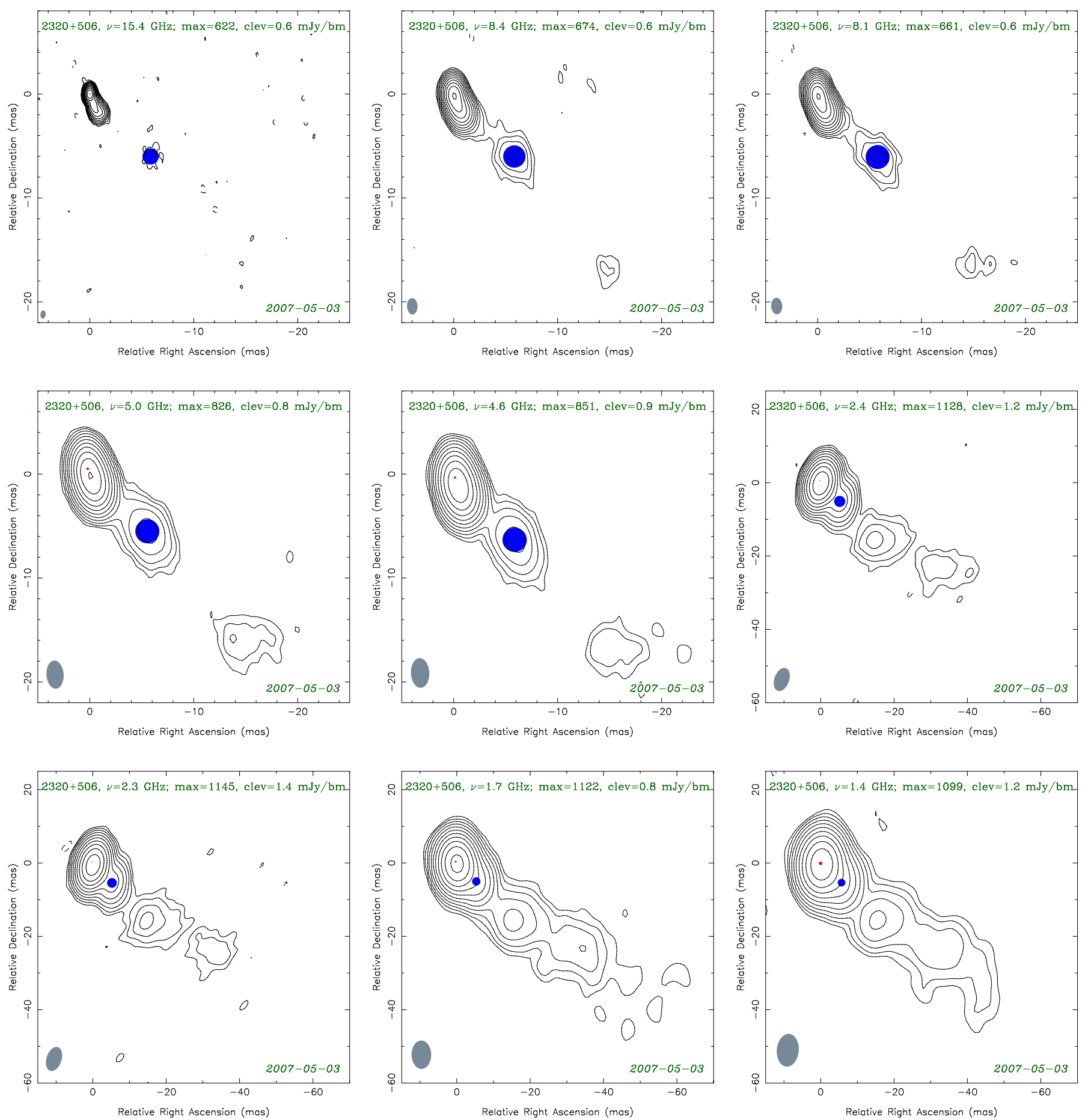

Fig. 1. continued. 